\title{
Warp Bridge Sampling: The Next Generation
}

\author{
Lazhi Wang ${ }^{1}$, David E. Jones ${ }^{2}$, and Xiao-Li Meng ${ }^{3 *}$ \\ Two Sigma Investments, LP ${ }^{1 \dagger}$ Texas A\&M University² ${ }^{2}$ and Harvard University ${ }^{3}$
}

June 11, 2019

\begin{abstract}
Bridge sampling is an effective Monte Carlo method for estimating the ratio of normalizing constants of two probability densities, a routine computational problem in statistics, physics, chemistry, and other fields. The Monte Carlo error of the bridge sampling estimator is determined by the amount of overlap between the two densities. In the case of uni-modal densities, Warp-I, II, and III transformations (Meng and Schilling, 2002) are effective for increasing the initial overlap, but they are less so for multi-modal densities. This paper introduces Warp-U transformations that aim to transform multi-modal densities into Unimodal ones without altering their normalizing constants. The construction of a Warp-U transformation starts with a Normal (or other convenient) mixture distribution $\phi_{\text {mix }}$ that has reasonable overlap with the target density $p$, whose normalizing constant is unknown. The stochastic transformation that maps $\phi_{\text {mix }}$ back to its generating distribution $\mathcal{N}(0,1)$ is then applied to $p$ yielding its Warp-U version, which we denote $\tilde{p}$. Typically, $\tilde{p}$ is unimodal and has substantially increased overlap with $\phi$. Furthermore, we prove that the overlap between $\tilde{p}$ and $\mathcal{N}(0,1)$ is guaranteed to be no less than the overlap between $p$ and $\phi_{\text {mix }}$, in terms of any $f$-divergence. We propose a computationally efficient method to find an appropriate $\phi_{\text {mix }}$, and a simple but effective approach to remove the bias which results from estimating the normalizing constants and fitting $\phi_{\text {mix }}$ with the same data. We illustrate our findings using 10 and 50 dimensional highly irregular multi-modal densities, and demonstrate how Warp-U sampling can be used to improve the final estimation step of the Generalized Wang-Landau algorithm (Liang, 2005), a powerful sampling and estimation method.
\end{abstract}

Keywords: Bridge sampling; Monte Carlo integration; Normalizing constants; Stochastic transformation; f-divergence; Normal mixture; Optimal transport; Wang-Landau algorithm

\section{Motivation}

Markov chain Monte Carlo (MCMC) methods, such as the Metropolis-Hastings algorithm, enable us to simulate from an unnormalized density without knowing its normalizing constant. However, in many scientific and statistical studies the very quantities of interest are normalizing constants or ratios of them; see, for example, Voter (1985), Kass and Raftery (1995), Meng and Wong (1996), DiCiccio et al. (1997), Gelman and Meng (1998), Shao and Ibrahim (2000), and Tan

${ }^{*}$ The authors gratefully acknowledge helpful conversations with members of the Department of Statistics at Harvard University, constructive comments from the audience of the 2016 MCQMC conference at Stanford University, and partial financial support from NSF and JTF. For correspondence, email david.jones@tamu.edu.

${ }^{\dagger}$ The views expressed herein are solely the views of the author(s) and are not necessarily the views of Two Sigma Investments, LP or any of its affiliates. They are not intended to provide, and should not be relied upon for, investment advice. Please see the full disclaimer on page 30. 
(2013). A well-known example from physics and chemistry is the computation of a partition function, which describes the statistical properties of a system in thermodynamic equilibrium. A partition function is the integral of an unnormalized density $q(\omega ; T, v)=\exp \{-H(\omega, v) /(k T)\}$, where $T$ is temperature, $k$ is Boltzmann's constant, $v$ is a vector of system characteristics, and $H(\omega, v)$ is the energy function. Because of the high dimensionality of $H$, Monte Carlo (MC) methods are often the only feasible tool for estimating a partition function, i.e., the normalizing constant of $q$; see Bennett (1976), Voter and Doll (1985), and Ceperley (1995).

Two key objects in statistics which can be expressed as normalizing constants are observeddata likelihoods and Bayes factors. Focusing on the latter, let $Y$ be our data, and let $M_{0}$ and $M_{1}$ be two plausible models parameterized by $\Theta_{0}$ and $\Theta_{1}$, respectively. The Bayes factor is then the ratio of the model likelihoods, $P\left(Y \mid M_{0}\right)$ and $P\left(Y \mid M_{1}\right)$, where

$$
P\left(Y \mid M_{i}\right)=\int P\left(Y \mid \Theta_{i}, M_{i}\right) P\left(\Theta_{i} \mid M_{i}\right) \mathbf{u}\left(\mathrm{d} \Theta_{i}\right)
$$

is the normalizing constant of the unnormalized density, $P\left(\Theta_{i}, Y \mid M_{i}\right) \propto P\left(Y \mid \Theta_{i}, M_{i}\right) P\left(\Theta_{i} \mid M_{i}\right)$, for $i=1,2$. In most Bayesian analyses, MC draws of $\Theta_{i}$ from $P\left(\Theta_{i} \mid Y, M_{i}\right)$ are made for the purpose of statistical inference, often using Monte Carlo Markov chain (MCMC) methods. Hence, to estimate $P\left(Y \mid M_{i}\right)$, it is desirable to use methods that require only these available draws (plus perhaps some draws from another convenient distribution).

One such method is the bridge sampling approach introduced by Bennett (1976) and generalized and popularized by Meng and Wong (1996). In this paper we propose an approach to improve the efficiency of bridge sampling in the multi-modal context, which is common when using even moderately complex models. Specifically, we introduce a class of stochastic transformations, Warp-U transformations, that can warp two multi-modal densities into densities having substantial overlap but without changing their respective normalizing constants. For bridge sampling, an increase in distributional overlap implies superior statistical efficiency. The key idea of Warp-U transformations is to approximate the unnormalized density of interest $q$ by a mixture distribution (e.g., a Normal mixture), and then to construct a coupling which allows us to (stochastically) map $q$ into a uni-modal density in the same way that the approximating mixture can be mapped back to a single generative density (e.g., a single Normal density). Our work builds on the warp transformations (centering, scaling, and symmetrizing) for uni-modal densities that were proposed by Meng and Schilling (2002). Our method also has an intrinsic connection with the theory of optimal transport (e.g. Villani, 2003), albeit here we typically only seek a reasonable transport (from one density to another) which can achieve a beneficial compromise between statistical efficiency and computational efficiency.

The utility of Warp- $U$ transformations is especially promising because bridge sampling is similar to many other mixture sampling approaches. Indeed, although developed from different perspectives, a number of methods in the literature turn out to be special cases of bridge 
sampling or adaptations of it, as demonstrated by Mira and Nicholls (2004). For instance, the marginal likelihood approaches of Chib (1995) and Chib and Jeliazkov (2001) based on Metropolis-Hastings output correspond to bridge sampling with specific choices of the bridge density. Similarly, the defensive sampling method of Hesterberg (1995) for estimating normalizing constants can be directly interpreted as bridge sampling. The 'balance weight heuristic' introduced by Veach and Guibas (1995) is a generalization of bridge sampling where the unnormalized densities to be integrated are not necessarily included among the sampling densities. This more general bridge sampling is also covered by the likelihood approach proposed by Kong et al. (2003), which reformulates MC integration as an estimation problem with the dominating measure as the estimand. Their likelihood framework provides a unified way of deriving and characterizing various strategies for boosting statistical efficiency, such as the control variates method of Owen and Zhou (2000); see Tan (2004), Meng (2005), and Kong et al. (2006) for details and illustrations. As discussed in Section 6.2, there is a possibility to cast Warp-U bridge sampling methods under the same likelihood framework and thereby make further connections with other methods, but that is a topic for future exploration.

Warp- $U$ transformations can additionally be useful in contexts where bridge sampling or analogous approaches are not applied or not initially applied. For example, Elvira et al. (2015) proposed a mixture sampling approach that, instead of using a mixture of all the sampling densities as MC weights, aims to save computation by using only some of the sampling densities to compute each weight. This is statistically less efficient than bridge sampling, but is based on the same ideas, and would also benefit from greater overlap between the sampling and target densities. For another example, consider the powerful adaptive importance sampling based methods which can be used to estimate normalizing constants, e.g., the multicanonical algorithm (Berg and Neuhaus, 1991), the $1 / k$-ensemble algorithm (Hesselbo and Stinchcombe, 1995), dynamic weighting (e.g., Wong and Liang, 1997; Liu et al., 2001), the Wang-Landau algorithm and adaptations of it (e.g., Wang and Landau, 2001; Liang, 2005; Liang et al., 2007; Bornn et al., 2013), and layered adaptive importance sampling (Martino et al., 2017). These are sampling methods, in contrast to (Warp-U) bridge sampling, which is a post-sampling method. Therefore, they are not directly comparable (e.g., costs for sampling and the final estimation are typically rather different). Instead, we show how Warp-U bridge sampling can be used as an additional step to improve adaptive importance sampling algorithms. Specifically, we illustrate that target draws can be obtained by re-sampling from the output of the Generalized Wang-Landau (GWL) algorithm (Liang, 2005), and that applying Warp-U bridge sampling to these draws can substantially reduce the mean squared error (MSE) of the final normalizing constant estimators.

A limitation of our work is that when we take into account computational cost Warp- $U$ bridge sampling is not necessarily superior to the following approach: first compute a mixture approximation to the target density exactly as in Warp-U bridge sampling, and then apply 
ordinary bridge sampling making use of this approximate density (see Section 3.3). However, Warp- $\mathrm{U}$ bridge sampling is a valuable development in that it fundamentally differs from this ordinary method and therefore provides new opportunities for improving computational efficiency in terms of precision per second. In particular, we anticipate that further work will yield approximations to Warp- $\mathrm{U}$ bridge sampling that enable it to surpass simpler methods based on ordinary bridge sampling, especially because here we have made no attempt to optimize our code or implementation; see the discussion in Section 6.

Our paper is organized as follows. Section 2 briefly overviews bridge sampling and the warp transformations of Meng and Schilling (2002), highlighting their power for increasing distribution overlap. Section 3 defines and illustrates the Warp-U transformation we propose and then establishes the theoretical result that Warp-U transformations never reduce distribution overlap. Section 4 outlines a computationally efficient strategy for finding a specific Warp-U transformation and studies the properties of the corresponding estimator. Section 5 demonstrates that the estimation performance of the Generalized Wang-Landau (GWL) algorithm introduced by Liang (2005) can be improved by combining it with Warp-U bridge sampling. Discussion is found in Section 6. The appendices in the online supplementary material provide a proof of Theorem 1, computational details, and guidance on selecting the tuning parameters of our method.

\section{The Basics of Warp Bridge Sampling}

Bridge sampling (Bennett, 1976; Meng and Wong, 1996) estimates the ratio of the normalizing constants of two unnormalized densities by leveraging the overlap between the two densities. Any method that can increase this overlap has the potential to reduce the MC error. The warp bridge sampling of Meng and Schilling (2002) explored this idea by transforming the original MC draws so that the densities of the transformed draws have substantially more overlap.

Let $q_{i}$ be an unnormalized density with normalizing constant $c_{i}$, for $i=1,2$. Furthermore, let $\mathbf{u}$ be the underlying measure common to both densities, typically the Lebesgue or counting measure. We use $p_{i}$ to denote the normalized density, i.e., $p_{i}(\omega)=c_{i}^{-1} q_{i}(\omega)$, for $\omega \in \Omega_{i}$, where $\Omega_{i}$ is the support of $q_{i}$. Our goal is to estimate the ratio $r=c_{1} / c_{2}$ or $\lambda=\log (r)$, using the draws, $\left\{w_{i, 1}, w_{i, 2}, \ldots, w_{i, n_{i}}\right\}$, from $p_{i}$, for $i=1,2$. In some instances, we only wish to estimate one normalizing constant $c_{1}$, in which case we will select $q_{2}=p_{2}$ to be a convenient density with $c_{2}=1$ (discussed in Section 3). Below, we begin by assuming that draws from $p_{1}$ and $p_{2}$ are given, but in Section 5 we combine our estimation strategy with the GWL sampling algorithm.

\subsection{Bridge Sampling}

Here we review the key aspects of bridge sampling that will be used in this paper. For a complete treatment, the reader is referred to Meng and Wong (1996) and the practical introduction by 
Gronau et al. (2017). An R package developed by Gronau et al. (2017) is available at https:

//cran.r-project.org/web/packages/bridgesampling/.

Bridge sampling relies on the fact that for any function, $\alpha$, defined on $\Omega_{1} \cap \Omega_{2}$ and satisfying $0<\left|\int_{\Omega_{1} \cap \Omega_{2}} \alpha(\omega) p_{1}(\omega) p_{2}(\omega) \mathbf{u}(\mathrm{d} \omega)\right|<\infty$, the following identity holds;

$$
r=\frac{c_{1}}{c_{2}}=\frac{\mathrm{E}_{2}\left[q_{1}(\omega) \alpha(\omega)\right]}{\mathrm{E}_{1}\left[q_{2}(\omega) \alpha(\omega)\right]},
$$

where $\mathrm{E}_{i}$ represents expectation with respect to $p_{i}$. Here $\alpha$ serves as a "bridge" connecting $p_{1}$ and $p_{2}$. The bridge sampling estimator of $r$ is the sample counterpart of (1), i.e.,

$$
\hat{r}_{\alpha}=\frac{n_{2}^{-1} \sum_{j=1}^{n_{2}} q_{1}\left(w_{2, j}\right) \alpha\left(w_{2, j}\right)}{n_{1}^{-1} \sum_{j=1}^{n_{1}} q_{2}\left(w_{1, j}\right) \alpha\left(w_{1, j}\right)} .
$$

For example, both importance sampling and geometric bridge sampling are special cases of bridge sampling, with $\alpha_{\text {imp }} \propto 1 / q_{2}$ and $\alpha_{\text {geo }} \propto 1 / \sqrt{q_{1} q_{2}}$, respectively.

Different choices of $\alpha$ lead to estimators with different statistical efficiency, which we quantify by the asymptotic variance of $\hat{\lambda}_{\alpha}=\log \left(\hat{r}_{\alpha}\right)$, or equivalently, the asymptotic relative variance of $\hat{r}_{\alpha}, \mathrm{E}\left(\hat{r}_{\alpha}-r\right)^{2} / r^{2}$. Under the assumption that all the $\mathrm{MC}$ draws used in (2) are i.i.d. (identically and independently distributed), Meng and Wong (1996) derived the first-order asymptotic variance of $\hat{\lambda}_{\alpha}$, from which they found that the optimal bridge has the form

$$
\alpha_{\mathrm{opt}}(\omega) \propto \frac{1}{s_{1} q_{1}(\omega)+r s_{2} q_{2}(\omega)}, \quad \text { where } s_{i}=\frac{n_{i}}{n}, i=1,2 .
$$

Before we proceed, we emphasize that the bridge sampling method itself does not require the assumption of i.i.d sampling; otherwise the method would be too limited to deserve a general $\mathrm{R}$ package. The i.i.d. assumption was invoked by Meng and Wong (1996) to make the theoretical calculation both feasible and insightful, in the sense that the resulting optimal bridge (3) takes an appealing mixture form which provides practical guidance. Indeed, regardless of whether the i.i.d. assumption holds, (3) provides a very effective bridge. In contrast, without the i.i.d. assumption the optimal bridge has a very involved expression (Romero, 2003), and offers little practical guidance. Therefore, for the rest of the article we invoke the i.i.d. assumption only for theoretical claims (e.g., when we refer to the "optimal" approach) or for simulation simplicity.

Because $\alpha_{\mathrm{opt}}$ depends on the unknown quantity $r$, Meng and Wong (1996) proposed an iterative sequence that rapidly converges to $\hat{r}_{\text {opt }}$, i.e.,

$$
\hat{r}_{\mathrm{opt}}^{(t+1)}=\frac{\frac{1}{n_{2}} \sum_{j=1}^{n_{2}}\left[\frac{l_{2, j}}{s_{1} l_{2, j}+s_{2} \hat{r}_{\mathrm{opt}}^{(t)}}\right]}{\frac{1}{n_{1}} \sum_{j=1}^{n_{1}}\left[\frac{1}{s_{1} l_{1, j}+s_{2} \hat{r}_{\mathrm{opt}}^{(t)}}\right]},
$$


where $l_{i, j}=q_{1}\left(w_{i, j}\right) / q_{2}\left(w_{i, j}\right)$, for $i=1,2$, and $j=1,2, \ldots, n_{i}$. Meng and Wong (1996) showed that, under the i.i.d. assumption, the asymptotic variance of $\hat{\lambda}_{\text {opt }}=\log \left(\hat{r}_{\text {opt }}\right)$ is

$$
\left(\frac{1}{n_{1}}+\frac{1}{n_{2}}\right)\left[\left(1-H_{\mathrm{A}}\left(p_{1}, p_{2}\right)\right)^{-1}-1\right]+o\left(\frac{1}{n_{1}+n_{2}}\right)
$$

which is the same as the asymptotic variance of the unobtainable optimal estimator $\hat{\lambda}_{\alpha_{\mathrm{opt}}}=$ $\log \left(\hat{r}_{\alpha_{\text {opt }}}\right)$. Here $H_{\mathrm{A}}\left(p_{1}, p_{2}\right)$ is the sample-size adjusted harmonic divergence between $p_{1}$ and $p_{2}$ :

$$
H_{\mathrm{A}}\left(p_{1}, p_{2}\right)=1-\int_{\Omega_{1} \cap \Omega_{2}}\left[w_{1} p_{1}^{-1}(\omega)+w_{2} p_{2}^{-1}(\omega)\right]^{-1} \mathbf{u}(\mathrm{d} \omega),
$$

with $w_{i}=s_{i}^{-1} /\left(s_{1}^{-1}+s_{2}^{-1}\right), i=1,2$. Using a likelihood that treats the baseline measure $\mathbf{u}$ as the (infinite dimensional) parameter, Kong et al. (2003) showed that $\hat{r}_{\mathrm{opt}}$ is the maximum likelihood estimator for $r$ (again, under the i.i.d. assumption), thereby further confirming its optimality.

\section{$2.2 \quad$ Warp Bridge Sampling}

For $i=1,2$, consider a transformation $\mathcal{F}_{i}$ of $w_{i, j}$ such that (a) the unnormalized density, $\tilde{q}_{i}$, of the transformed draws, $\tilde{w}_{i, j}=\mathcal{F}_{i}\left(w_{i, j}\right)$, has the same normalizing constant as $q_{i}$, and (b) $H_{\mathrm{A}}\left(\tilde{p}_{1}, \tilde{p}_{2}\right)<H_{\mathrm{A}}\left(p_{1}, p_{2}\right)$. Then by $(5)$, the optimal bridge sampling estimator based on the transformed draws $\left\{\left(\tilde{w}_{i, 1}, \ldots, \tilde{w}_{i, n_{i}}\right) ; i=1,2\right\}$ will have smaller asymptotic variance than that based on the original draws $\left\{\left(w_{i, 1}, \ldots, w_{i, n_{i}}\right) ; i=1,2\right\}$, assuming the draws are independent. This observation motivated the Warp transformations proposed by Meng and Schilling (2002), whose contribution also demonstrated empirically the benefit of Warp transformations under general MCMC settings (i.e., without requiring i.i.d. draws).

The simple idea of Warp-I transformations is to increase overlap among densities (e.g., in terms of $H_{A}$ in (6)) by shifting them so that they share a common location. Specifically, let $\mu_{i}$ be a location parameter (e.g., mean or mode) of $p_{i}$, for $i=1,2$, and suppose that the dominating measure (e.g., the Lebesgue measure) is invariant to translation. Let $\tilde{w}_{i, j}^{(\mathrm{I})}=w_{i, j}-\mu_{i}$ and denote the corresponding unnormalized density by $\tilde{q}_{i}^{(\mathrm{I})}(w)=q_{i}\left(w+\mu_{i}\right)$; clearly this density has the same normalizing constant $c_{i}$ as the original target $q_{i}(w)$, for $i=1,2$. The Warp-I bridge sampling estimator is then obtained by replacing $w_{i, j}$ and $q_{i}$ in $(2)$ with $\tilde{w}_{i, j}^{(\mathrm{I})}$ and $\tilde{q}_{i}^{(\mathrm{I})}$, respectively.

The next obvious transformation is to match both the location and the spread. Let $\mu_{i}$ be a location parameter and $\mathcal{S}_{i}$ be a scaling parameter, for $i=1,2$. The Warp-II transformation then sets $\tilde{w}_{i, j}^{(\mathrm{II})}=\mathcal{S}_{i}^{-1}\left(w_{i, j}-\mu_{i}\right)$ and $\tilde{q}_{i}^{(\mathrm{II})}(\omega)=\left|\mathcal{S}_{i}\right| q_{i}\left(\mathcal{S}_{i} \omega+\mu_{i}\right)$. The dash-dot curve in the left panel of Figure 1 illustrates that $\tilde{p}_{1}^{(\mathrm{II})}$ overlaps more with $p_{2}$ than $p_{1}$ does. It also overlaps more than the Warp-I transformed density $\tilde{p}_{1}^{(\mathrm{I})}$ does (not shown).

Warp-III transformations increase overlap further by making the densities in question symmetric via a stochastic transformation. Specifically, a Warp-III transformation sets $\tilde{w}_{i, j}^{(\text {III) }}=$ $\xi_{j} \mathcal{S}_{i}^{-1}\left(w_{i, j}-\mu_{i}\right)$, where $\xi_{j}$ takes on the value 1 or -1 with equal probability (independently of $\left.w_{i, j}\right)$. The unnormalized density of $\tilde{w}^{(\mathrm{III})}$ is $\tilde{q}_{i}^{(\mathrm{III})}(\omega)=\left|\mathcal{S}_{i}\right|\left[q_{i}\left(\mu_{i}-\mathcal{S}_{i} \omega\right)+q_{i}\left(\mu_{i}+\mathcal{S}_{i} \omega\right)\right] / 2$, an 

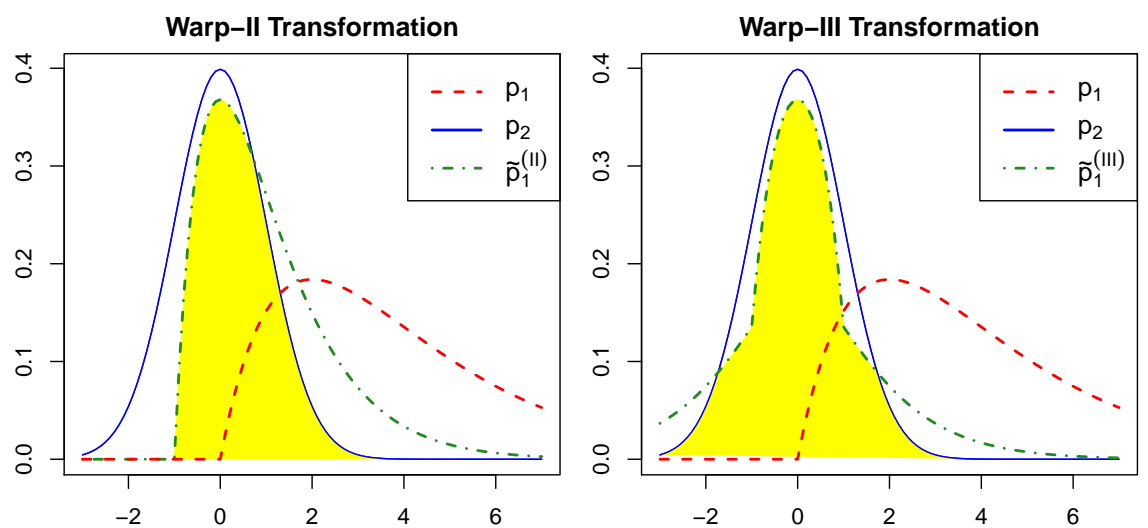

Figure 1: Graphical illustration of Warp-II (left) and Warp-III transformations (right). The dashed and the solid lines are the curves of $p_{1}$ and $p_{2}$. The dash-dot lines are $p_{1}^{(\mathrm{II})}$ (left) and $p_{1}^{(\mathrm{III})}$ (right), obtained by Warp-II and Warp-III transformation, respectively. The shaded areas highlight the much increased overlap between the warp-transformed densities and the reference density $p_{2}=N(0,1)$.

example of which is shown in the right panel of Figure 1 (dash-dot curve). Below we show that stochastic transformations are also very powerful in dealing multi-modality, a challenging issue in MC based estimation and indeed in statistical inference more generally.

\section{$3 \quad$ Warp-U Bridge Sampling}

Consider a unimodal density, $\phi$, such as a $\mathcal{N}\left(0, I_{d}\right)$ or $t$-distribution. The key idea of our approach is to construct a stochastic transformation of the original MC draws such that the density for the transformed draws is much closer to $\phi$. To simplify the exposition, we consider the problem of estimating a single normalizing constant and fix the other density used in the bridge sampling estimator (2) to be $\phi$. The problem of estimating a ratio of two normalizing constants can then be handled in the following two ways. Firstly, we can use two bridge sampling estimators, one in the numerator and one in the denominator, based on the Warp- $\mathrm{U}$ transformed draws $\left\{\tilde{w}_{i, 1}, \ldots, \tilde{w}_{i, n_{i}}\right\} \sim \tilde{p}_{i}$ and draws from the convenient unimodal auxiliary distribution $\left\{z_{i, 1}, \ldots, z_{i, m_{i}}\right\} \sim \phi$, for $i=1,2$ respectively. The two estimators can share the same auxiliary distribution $\phi$, or even the same set of auxiliary draws. We emphasize again here that we do not require any of these draws to be i.i.d., though typically those from the auxiliary distribution are i.i.d. because it is usually easy to obtain i.i.d. draws from $\phi$. Secondly, we could disregard $\phi$ after the warp transformation and then use one bridge sampling estimator of the ratio $r$ based only on the full set of the transformed draws $\bigcup_{i=1}^{2}\left\{\tilde{w}_{i, 1}, \ldots, \tilde{w}_{i, n_{i}}\right\}$. This second strategy is effective because if $\tilde{p}_{1}$ and $\tilde{p}_{2}$ both overlap significantly with $\phi$ then they are likely to also have substantial overlap with each other. 
Since we focus on a single unnormalized density $q$, we drop the double indices and let $\left\{w_{1}, \ldots, w_{n}\right\}$ be $n$ draws from $p=c^{-1} q$, where $p$ is assumed to be a continuous density on $\mathbb{R}^{d}$. Similarly, we use $\left\{z_{1}, \ldots, z_{m}\right\}$ to denote $m$ i.i.d. draws from $\phi$. For concreteness, we set $\phi=\mathcal{N}\left(0, I_{d}\right)$, but other choices of $\phi$ can work equally well or even better.

\subsection{Constructing Warp-U Transformations}

When $q$ is multi-modal, we could approximate it by a Gaussian mixture $\phi_{\text {mix }}$ and then perform standard bridge sampling using $q$ and $\phi_{\text {mix. }}$. Warp-U bridge sampling aims to improve on this approach but begins in the same way. Let

$$
\phi_{\text {mix }}(x ; \boldsymbol{\zeta})=\sum_{k=1}^{K} \phi^{(k)}(x)=\sum_{k=1}^{K} \pi_{k}\left|\mathcal{S}_{k}\right|^{-1} \phi\left(\mathcal{S}_{k}^{-1}\left(x-\mu_{k}\right)\right),
$$

where $\phi^{(k)}$ represents the $k$-th component in $\phi_{\text {mix }}$, including its weight $\pi_{k}$, for $k=1, \ldots, K$, and $\boldsymbol{\zeta}$ collects the transformation parameters $\bigcup_{k=1}^{K}\left\{\pi_{k}, \mu_{k}, \mathcal{S}_{k}\right\}$. Alspach and Sorenson (1972) showed that any piecewise continuous density can be approximated arbitrarily well by a Gaussian mixture of the form (7) as $K \rightarrow \infty$ (specifically, they demonstrated uniform convergence). In practice, for a reasonable choice of $K$, it is usually possible to find a $\phi_{\text {mix }}$ that has substantial overlap with $p$. Section 4 will discuss how to estimate $\phi_{\text {mix }}$. Here we assume that $\phi_{\text {mix }}$ is known in order to describe the Warp-U transformation itself.

The Warp- $U$ transformation uses a coupling between augmented random variables drawn from $\phi_{\text {mix }}$ and $p$, and we now specify this relationship. Suppose $X \sim \phi_{\text {mix }}$, depicted in Figure 2(a) as the solid line, then we can write $X=\mathcal{S}_{\Theta} Z+\mu_{\Theta}$, where $Z \sim \phi$ and is independent of $\Theta$, a discrete random variable distributed such that $P(\Theta=k)=\pi_{k}$ for $k=1, \ldots, K$. Figure 2(b) shows the joint distribution of $\Theta$ and $X$, with their marginal distributions on the two faded vertical plates. The random index $\Theta$ induces a random transformation

$$
\mathcal{F}_{\Theta}(x)=\mathcal{S}_{\Theta}^{-1}\left(x-\mu_{\Theta}\right) .
$$

It follows trivially that if we draw $(x, \theta)$ from the joint distribution of $(X, \Theta)$, then $\tilde{x}=\mathcal{F}_{\theta}(x) \sim \phi$.

Next, let $W$ be a random variable from $p$ and $\Psi$ be a random index. We create a coupling between $(W, \Psi)$ and $(X, \Theta)$ by requiring that $\Psi \mid W$ and $\Theta \mid X$ have the same distribution, i.e.,

$$
\varpi(k \mid \omega) \triangleq P(\Psi=k \mid W=\omega) \equiv P(\Theta=k \mid X=\omega)=\phi^{(k)}(\omega) / \phi_{\text {mix }}(\omega), \quad k=1, \ldots, K .
$$

We can then decompose $p$ into $K$ components, i.e., $p(\omega)=\sum_{k=1}^{K} p^{(k)}(\omega)$, where

$$
p^{(k)}(\omega)=p(\omega, \Psi=k)=p(\omega) \frac{\phi^{(k)}(\omega)}{\phi_{\text {mix }}(\omega)} .
$$

Figure 2(c) shows the joint distribution of $(W, \Psi)$ (thick dashed curves) and their marginal distributions (thin dash curves in the two vertical plates). The Warp-U transformation is then 
(a)

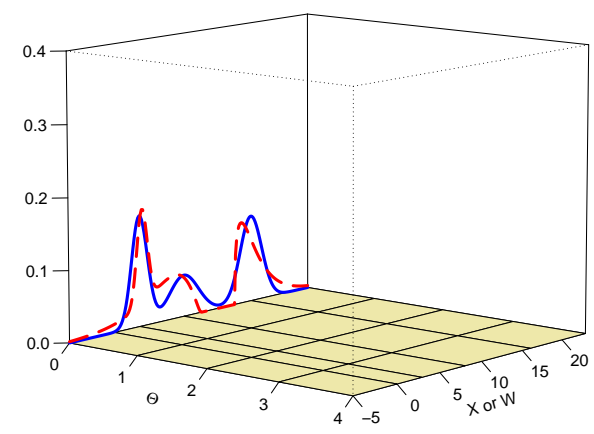

(c)

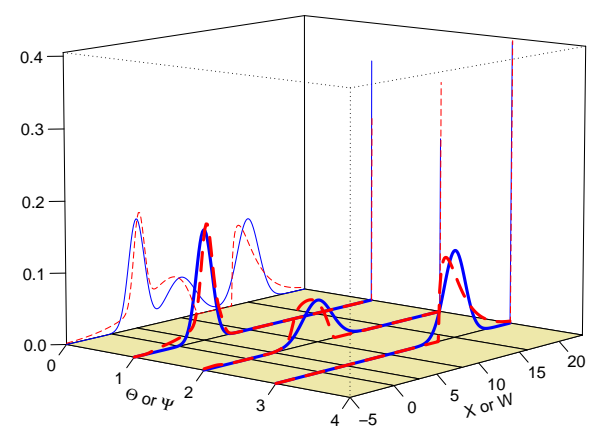

(b)

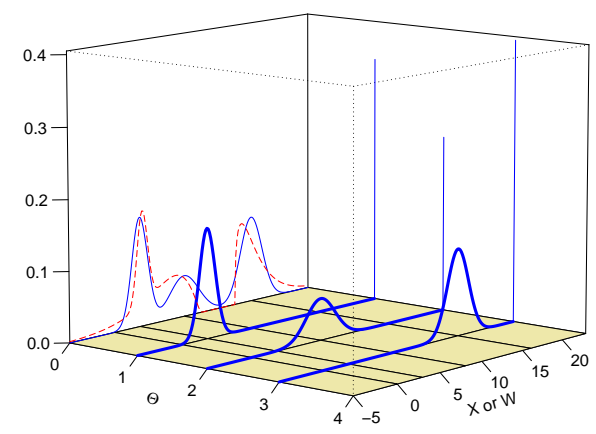

(d)

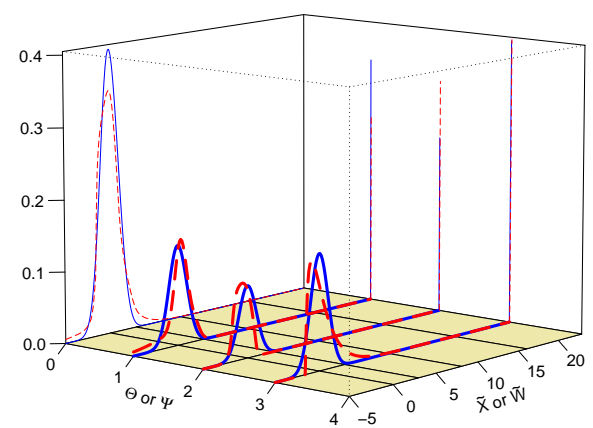

Figure 2: Illustration of Warp-U transformation. (a) $\phi_{\text {mix }}$ (solid line) and $p$ (dashed line); (b) the joint and marginal distributions of $X$ and $\Theta$ (solid line); (c) the joint and marginal distributions of $W$ and $\Psi$ (dashed line); (d) the joint and marginal distributions of $\Theta$ and $\widetilde{X}$ (solid line) and those of $\Psi$ and $\widetilde{W}$ (dashed line), where $\widetilde{X}$ and $\widetilde{W}$ are obtained via Warp-U transformation.

constructed by again using the map in (8) but with $(W, \Psi)$ in place of $(X, \Theta)$,

$$
\widetilde{W}=\mathcal{F}_{\Psi}(W)=\mathcal{S}_{\Psi}^{-1}\left(W-\mu_{\Psi}\right) \sim \widetilde{p} .
$$

Intuitively, because the transformation $\mathcal{F}$ maps the multi-modal $\phi_{\text {mix }}$ back to the original unimodal (generating) density $\phi$, when it is applied to the multi-modal $p$, it can achieve similar a "uni-modalizing" effect because $\phi_{\text {mix }}$ was chosen to approximate $p$.

In practice, to apply a Warp-U transformation to $w_{j}$, we calculate $\varpi\left(\cdot \mid w_{j}\right)$ according to $(9)$, draw $\psi_{j}$ from $\varpi\left(\cdot \mid w_{j}\right)$, and finally apply the deterministic transformation $\mathcal{F}_{\psi_{j}}$ to $w_{j}$. Graphically, each $p^{(k)}$ in Figure 2(c) is re-centered and re-scaled, like its counterpart, $\phi^{(k)}$. The dashed lines in Figure 2(d) are the joint distribution of $\Psi$ and the Warp-U transformed variable, $\widetilde{W}$. In the faded left vertical panel of $2(\mathrm{~d})$, we see that the distribution of $\widetilde{W}$ overlaps substantially with $\phi$.

When $K=1$, the Warp- $\mathrm{U}$ transformation is the same as the Warp-II transformation provided that we choose $\phi_{\text {mix }}$ to be a location-scale family. For $K>1$, Theorem 1 in Section 3.2 (below) ensures that there will be additional overlap between $\tilde{p}$ and $\phi$ compared to the overlap between $p$ and $\phi_{\text {mix }}$, except for in trivial cases, e.g., when $p=\phi_{\text {mix }}$. 


\subsection{Theoretical Guarantee for General Warp-U Transformations}

Figure 3 summarizes the key variables and distributions underlying a general Warp-U transformation, which does not assume that $\phi$ is the Normal density. We do still require that $\phi$ shares the same support $\Omega$ as our target $p$. Another generalization included in Figure 3 is that the "index variable" $\Theta$ (and hence also $\Psi$ ) is permitted to take on any distribution $\pi$ with support $\Pi$ and dominating measure $\mathbf{v}$, and in particular $\Theta$ (and $\Psi$ ) is no longer required to be discrete.

For all $\theta \in \Pi$, the map $\mathcal{F}_{\theta}$ in Figure 3 is required to be one-to-one, onto, and almost surely differentiable, and to satisfy $\Omega=\mathcal{F}_{\theta}(\Omega)$. We denote its inverse map by $\mathcal{H}_{\theta}$. Since we specify $X \sim \mathcal{H}_{\theta}(Z)$, where $Z \sim \phi$, the conditional distribution $X \mid \Theta=\theta$ is

$$
\phi_{X \mid \Theta}(x \mid \theta)=\phi\left(\mathcal{F}_{\theta}(x)\right)\left|\mathcal{F}_{\theta}^{\prime}(x)\right|, \quad x \in \Omega
$$

and the (marginal) density of $X$ is

$$
\phi_{\text {mix }}(x)=\int_{\Pi} \phi_{X \mid \Theta}(x \mid \theta) \pi(\theta) \mathbf{u}(\mathrm{d} \theta)=\int_{\Pi} \phi\left(\mathcal{F}_{\theta}(x)\right)\left|\mathcal{F}_{\theta}^{\prime}(x)\right| \pi(\theta) \mathbf{u}(\mathrm{d} \theta) .
$$

Let $\varpi(\cdot \mid x)$ be the conditional distribution $\Theta \mid X=x$,

$$
\varpi(\theta \mid x)=\frac{\phi_{X \mid \Theta}(x \mid \theta) \pi(\theta)}{\phi_{\text {mix }}(x)}, \quad \theta \in \Pi,
$$

and, as before, let the variable $\Psi$ be defined through $P(\Psi=\theta \mid W=\omega)=\varpi(\theta \mid \omega)$. The joint distributions of $(\Psi, W)$ and $(\Theta, X)$ therefore share the same conditional specification:

$$
p_{\Psi, W}(\theta, \omega)=\varpi(\theta \mid \omega) p(\omega) \quad \text { and } \quad \phi_{\Theta, X}(\theta, \omega)=\varpi(\theta \mid \omega) \phi_{\text {mix }}(\omega), \quad(\omega, \theta) \in \Omega \times \Pi .
$$

Considering this shared structure, here and in what follows we sometimes use the dummy variables $(\omega, \theta)$ to refer to realizations of both $(W, \Psi)$ and $(X, \Theta)$, and prevent confusion through our notation for the density functions in question.

The key consequence of the coupling (15) is that the overlap between $\phi$ and the density of the Warp-U transformed $W: \widetilde{W}=\mathcal{F}_{\Psi}(W) \sim \tilde{p}$, is greater than that between $\phi_{\text {mix }}$ and $p$. To prove this mathematically, we need a measure or multiple measures of overlap. The notion of $f$-divergence, or more precisely its complement (since small divergence corresponds to large overlap), serves well for our purposes. For any (non-trivial) convex function $f$ on $[0, \infty)$ such that $f(1)=0$, the corresponding $f$-divergence between two probability densities $p_{1}$ and $p_{2}$, when $p_{1}$ is absolutely continuous with respect to $p_{2}$, is defined as

$$
\mathcal{D}_{f}\left(p_{1} \| p_{2}\right)=\int_{\Omega} p_{2}(\omega) f\left(\frac{p_{1}(\omega)}{p_{2}(\omega)}\right) \mathbf{u}(\mathrm{d} \omega) .
$$

Theorem 1 below states that Warp- $U$ transformations reduce any $f$-divergence, unless either the transformation or $f$ is trivially chosen, in which cases the $f$-divergence is unchanged. The proof is given in Appendix A.

Theorem 1. Let the Warp-U transformation $\mathcal{F}_{\Psi}$ be defined as in Figure 3, with the conditions given in the caption. The following results then hold. 


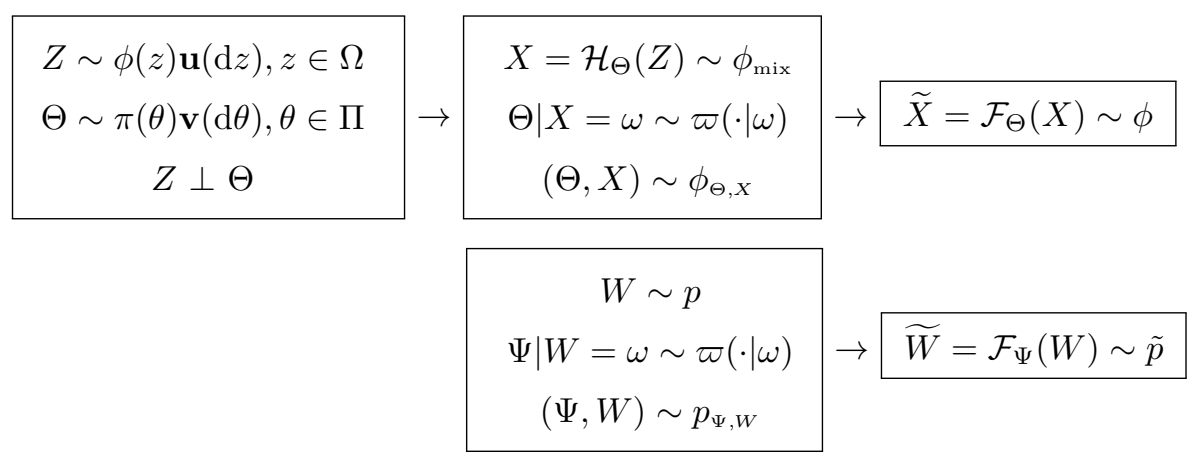

Figure 3: Relationships among the random variables and their distributions for Warp-U transformation. Here for almost surely (with respect to $\mathbf{v}$ ) all values of $\theta \in \Pi, \mathcal{F}_{\theta}$ and its inverse $\mathcal{H}_{\theta}$ are one-to-one, onto, and almost surely (with respect to $\mathbf{u}$ ) differentiable maps from $\Omega \rightarrow \Omega$.

(I) For any $f$-divergence $\mathcal{D}_{f}$, we have $\mathcal{D}_{f}(\tilde{p} \| \phi) \leqslant \mathcal{D}_{f}\left(p \| \phi_{\text {mix }}\right)$.

(II) If $f$ is strictly convex, then the equality in (I) holds if and only if $\ell(\theta ; \tilde{\omega}) \equiv \frac{p\left(\mathcal{H}_{\theta}(\tilde{\omega})\right)}{\phi_{\operatorname{mix}}\left(\mathcal{H}_{\theta}(\tilde{\omega})\right)}$ is free of $\theta$ (almost surely with respect to $\mathbf{v} \times \mathbf{u}$ ).

The Hellinger distance, the weighted harmonic divergence in (6), and the $L_{1}$ distance are all $f$-divergences, with $f_{H e}(t)=0.5(1-\sqrt{t})^{2}, f_{H a}(t)=w_{1}(1-t) /\left(w_{1}+w_{2} t\right)$, and $f_{L_{1}}(t)=|1-t|$, respectively. The weighted harmonic divergence in (6) is an especially important case because it determines the asymptotic variance of bridge sampling estimators (see (5)). Consequently, Theorem 1 says that the bridge sampling estimator based on $\tilde{p}$ and $\phi$ has smaller asymptotic variance than that based on $p$ and $\phi_{\text {mix }}$, thus supporting the use of Warp- $\mathrm{U}$ transformations (Section 3.3 below gives the explicit form of these two estimators). Interestingly, inequality (I) does not necessarily hold for $L_{\mathrm{p}}$ distance when $\mathrm{p} \neq 1$ (and hence $L_{\mathrm{p}}$ distance is not an $f$-divergence when $\mathrm{p} \neq 1$ ). As a simple counter-example, let $K=1$ in (7) and therefore $\phi_{\text {mix }}(\omega)=|\mathcal{S}|^{-1} \phi\left(\mathcal{S}^{-1}(\omega-\mu)\right)$. Then $\tilde{p}(\omega)=|\mathcal{S}| p(\mathcal{S} \omega+\mu)$, and

$$
L_{\mathrm{p}}(\tilde{p}, \phi)=\left(\int|| \mathcal{S}|p(\mathcal{S} \tilde{\omega}+\mu)-\phi(\tilde{\omega})|^{\mathrm{p}} \mathbf{u}(\mathrm{d} \tilde{\omega})\right)^{\mathrm{p}^{-1}}=|\mathcal{S}|^{1-\mathrm{p}^{-1}} L_{\mathrm{p}}\left(p, \phi_{\text {mix }}\right),
$$

so $L_{\mathrm{p}}(\tilde{p}, \phi)>L_{\mathrm{p}}\left(p, \phi_{\text {mix }}\right)$ whenever $|\mathcal{S}|^{1-\mathrm{p}^{-1}}>1\left(\right.$ and $\left.L_{\mathrm{p}}\left(p, \phi_{\text {mix }}\right)>0\right)$.

Part (II) of Theorem 1 means that a Warp-U transformation will always result in real gain, as measured by any strictly convex $f$-divergence, unless one of two situations occur: (A) $\phi_{\text {mix }}$ is a perfect fit to $p$, in which case obviously $\ell(\theta, \tilde{\omega})=1$; or (B) $p \neq \phi_{\text {mix }}$, but the Warp-U transformation $\mathcal{F}_{\Theta}$ is unfortunately (or unwisely) chosen such that it renders the "likelihood ratio" $\ell(\theta ; \tilde{\omega})$ flat as a function of $\theta$. Situation (B) includes the trivial cases where $\mathcal{F}_{\theta}$ does not depend on $\theta$, or $\theta$ does not vary because $\pi$ is a singleton, as well as some more complex scenarios.

An illustration of Theorem 1 is given in Figure 4 and Table 1 for the case of a tri-modal target distribution $p$ and an approximating density $\phi_{\text {mix }}$ with $K=2$. The green region in Figure 

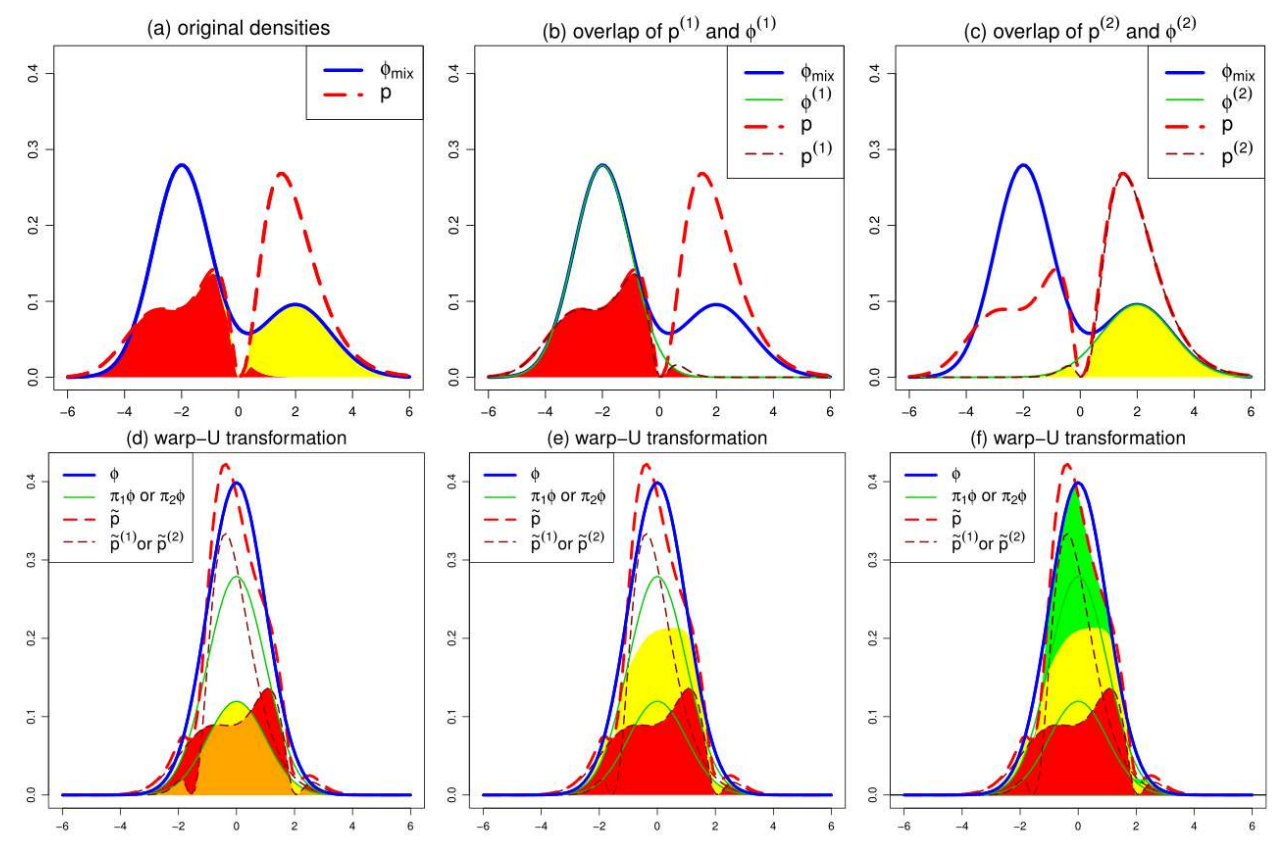

Figure 4: Illustration of the increase in the area of the overlapping region after Warp-U transformation. (a) $p$ (dashed line) and $\phi_{\text {mix }}$ (solid line); (b) the 1st component of $p$, denoted as $p^{(1)}$ (thin dashed line), the 1st component of $\phi_{\text {mix }}$, denoted as $\phi^{(1)}$ (thin solid line), and their overlap (shaded in red); (c) $p^{(2)}$, $\phi^{(2)}$, and their overlap (shaded in yellow); (d) the corresponding curves and shaded areas after Warp-U transformation; (e) the yellow region is added on top of the red region; (f) the green area shows the additional cross overlap between the 1st and 2nd components induced by the Warp-U transformation.

$4(\mathrm{f})$ shows that the final overlap between $\tilde{p}$ and $\phi$ after Warp- $\mathrm{U}$ transformation is greater than the sum of the overlaps between $p^{(k)}$ and $\phi^{(k)}$, for $k=1,2$. We call the additional overlap cross-overlap, and it is this phenomenon which is key to Warp-U transformations. Table 1 lists several $f$-divergences for the pairs $\left(p, \phi_{\text {mix }}\right)$ and $(\tilde{p}, \phi)$, and it confirms that the $f$-divergences are much lower in the latter case. Indeed, due to cross-overlap, the overlapping area for the pair of densities $(\tilde{p}, \phi)$ is nearly $40 \%$ larger than that for $\left(p, \phi_{\text {mix }}\right)$.

Table 1: The overlapping area, and the distances between $p$ and $\phi_{\text {mix }}$ and between $\tilde{p}$ and $\phi$.

\begin{tabular}{|l|c|c|c|c|}
\hline Densities & Overlap area & $L_{1}$ distance & Hellinger distance & harmonic divergence \\
\hline$\left(p, \phi_{\text {mix }}\right)$ & 0.66 & 0.68 & 0.28 & 0.145 \\
\hline$(\tilde{p}, \phi)$ & 0.92 & 0.16 & 0.08 & 0.013 \\
\hline
\end{tabular}




\subsection{Warp-U Bridge Sampling}

After the parameters $\zeta$ for $\phi_{\text {mix }}$ have been chosen, the Warp- $U$ transformation is determined. The unnormalized density of the transformed draws $\left\{\tilde{w}_{1}, \ldots, \tilde{w}_{n}\right\}$ can then be expressed as

$$
\tilde{q}(\tilde{w} ; \boldsymbol{\zeta})=\sum_{k=1}^{K} c p^{(k)}\left(\omega=\mathcal{S}_{k} \tilde{w}+\mu_{k}\right)=\phi(\tilde{w}) \sum_{k=1}^{K} \frac{q\left(\mathcal{S}_{k} \tilde{w}+\mu_{k}\right)}{\phi_{\text {mix }}\left(\mathcal{S}_{k} \tilde{w}+\mu_{k}\right)} \pi_{k} .
$$

Clearly, the normalizing constants of $\tilde{q}$ and $q$ are both $c$, and hence we can estimate $c$ with the bridge sampling estimator based on $\left\{\tilde{w}_{1}, \ldots, \tilde{w}_{n}\right\} \sim \tilde{p}$ and $\left\{z_{1}, \ldots, z_{m}\right\} \sim \phi$, i.e.,

$$
\hat{c}_{\alpha}^{(\mathrm{U})} \equiv \hat{r}_{\alpha}^{(\mathrm{U})}=\frac{m^{-1} \sum_{j=1}^{m} \tilde{q}\left(z_{j} ; \boldsymbol{\zeta}\right) \alpha\left(z_{j} ; \tilde{p}, \phi\right)}{n^{-1} \sum_{j=1}^{n} \phi\left(\tilde{w}_{j}\right) \alpha\left(\tilde{w}_{j} ; \tilde{p}, \phi\right)} .
$$

As mentioned in Section 2.1, the optimal choice of $\alpha(\cdot ; \tilde{p}, \phi)$ is proportional to $\left(s_{1} \tilde{p}+s_{2} \phi\right)^{-1}$. Since $\phi_{\text {mix }}$ also has some overlap with $p$, the normalizing constant can alternatively be estimated with the bridge sampling estimator based on $\left\{w_{1}, \ldots, w_{n}\right\} \sim p$ and $\left\{x_{1}, \ldots, x_{m}\right\} \sim \phi_{\text {mix }}$, i.e.,

$$
\hat{c}_{\alpha}^{(\text {mix })} \equiv \hat{r}_{\alpha}^{(\text {mix })}=\frac{m^{-1} \sum_{j=1}^{m} q\left(x_{j}\right) \alpha\left(x_{j} ; p, \phi_{\text {mix }}\right)}{n^{-1} \sum_{j=1}^{n} \phi_{\text {mix }}\left(w_{j} ; \zeta\right) \alpha\left(w_{j} ; p, \phi_{\text {mix }}\right)} .
$$

Theorem 1 implies $\mathcal{D}(\tilde{p}, \phi) \leqslant \mathcal{D}\left(p, \phi_{\text {mix }}\right)$ when $\mathcal{D}$ is the weighted harmonic divergence in (6), so the asymptotic variance of $\hat{\lambda}_{\alpha}^{(\mathrm{U})}=\log \left(\hat{c}_{\alpha}^{(\mathrm{U})}\right)$ is smaller than that of $\hat{\lambda}_{\alpha}^{(\text {mix })}=\log \left(\hat{c}_{\alpha}^{(\text {mix })}\right)$ under the optimal choice of $\alpha$, when the draws are independent. Even when we choose some other $\alpha$ (e.g., the geometric mean $\sqrt{p_{1} p_{2}}$ ) or the draws are not independent, we can still expect that the increased overlap obtained by the Warp-U transformation helps (18) to outperform (19), at least when both use the same $n$ and $m$.

In challenging situations, our choice of $\phi_{\text {mix }}$ may be a poor match with $p$. This will clearly impact the quality of the corresponding Warp-U transformation, but $\mathcal{D}(\tilde{p}, \phi) \leqslant \mathcal{D}\left(p, \phi_{\text {mix }}\right)$ will nevertheless still hold. Perhaps the most important case where the Warp-U transformation is not beneficial is when $p$ has many highly isolated modes. The cross-overlap discussed in Section 3.2 will then be small, and so will the reduction achieved by $\mathcal{D}(\tilde{p}, \phi)$. Consequently, in this scenario, the extra computation required by the Warp- $\mathrm{U}$ transformation is not worthwhile.

\section{Warp-U Computation Details and Numerical Examples}

The key step in applying Warp-U bridge sampling is to identify a mixture density $\phi_{\text {mix }}$ that adequately overlaps with $p$, under reasonable constraints on computation. In relatively lowdimensional $(\leqslant 10)$ problems, we can obtain $\phi_{\text {mix }}$ based on the expression for $q$, e.g., using iterated Laplace approximations (see Bornkamp, 2011; Gelman et al., 2013). However, these methods are too costly and unstable in high dimensions. Below we outline a simple method which uses the draws $\left\{w_{1}, \ldots, w_{n}\right\}$, can capture a good proportion of the mass of $p$, and has computational cost that is linear in dimensionality. We then adopt another practical strategy to remove an over-fitting bias due to this simple method. 


\subsection{Fitting $\phi_{\text {mix }}$ : Diagonal Covariance Matrices}

Suppose that $p$ is $D$ dimensional and that our draws from $p$ reasonably represent the regions of non-negligible density. We seek a Normal mixture $\phi_{\text {mix }}$ in the form of (7) to approximate $p$, where $\mathcal{S}_{k}$ is a positive definite diagonal matrix, $\mathcal{S}_{k}=\operatorname{Diag}\left\{\sigma_{k, 1}, \sigma_{k, 2}, \ldots, \sigma_{k, D}\right\}$, for $k=1, \ldots, K$, and hence $\boldsymbol{\zeta}=\left(\pi_{1}, \ldots, \pi_{K}, \mu_{1}, \ldots, \mu_{K}, \mathcal{S}_{1}, \ldots, \mathcal{S}_{K}\right)$. Unlike usual statistical inference problems where ignoring correlations can have very serious consequences, for Warp- $U$ transformations using diagonal covariance matrices is often an acceptable compromise between computational efficiency and MC efficiency. Indeed, as discussed in Section 3, it is not necessary for $\phi_{\text {mix }}$ to be a great fit to $p$ in order for us to benefit significantly from Warp-U transformations. In the next section, we provide further empirical evidence to illustrate this point.

Since a mixture of Normal components without suitable restrictions has unbounded likelihood (Kiefer and Wolfowitz, 1956; Day, 1969), we estimate $\boldsymbol{\zeta}$ by the penalized MLE proposed by Chen et al. (2008). In particular, we make use of the EM procedure proposed by Chen and Tan (2009), but with a "robustified" penalty function

$$
\mathbf{p}_{\mathbf{n}}(\boldsymbol{\zeta})=-\frac{1}{\sqrt{n}} \sum_{k=1}^{K} \sum_{d=1}^{D}\left\{\frac{\widehat{I Q}_{d}^{2}}{\sigma_{k, d}^{2}}-\log \left(\sigma_{k, d}^{2}\right)\right\},
$$

where $\widehat{I Q}_{d}$ is the inter-quantile range of the draws from $p$ in the $d$-th dimension. Because EM tends to become trapped at local modes, we apply it $M$ times, randomly generating a new initial point $\boldsymbol{\zeta}^{(0)}$ for each repetition as follows. The initial values for the $\pi_{k}$ 's and $\mathcal{S}_{k}$ 's are $\pi_{k}^{(k)}=K^{-1}$ and $\sigma_{k, d}^{2}=1.5 \widehat{I Q}_{d}^{2}$ for all $k$ and all $M$ replications. For the mean parameters $\mu_{k}$, for the first $M / 2$ replications, we randomly sample $K$ of available draws from $p$ (without replacement) to be the initial values. For the second $M / 2$ replications, along the dimension with the largest estimated variance, we first identify a region where $95 \%$ of the draws from $p$ reside and divide it into $K$ subregions so that each subregion contains approximately the same number of draws. We then sample one draw from each of the $K$ subregions to set the initial mean parameters. Our EM stopping criterion is $\left|1-\left(l_{n}^{(t)} / l_{n}^{(t-1)}\right)\right|<10^{-6}$, where $l_{n}^{(t)}$ is the value of the (un-penalized) $\log$-likelihood at iteration $t$. In our simulations, the EM usually stopped within 100 iterations. After obtaining $M$ estimates of $\boldsymbol{\zeta}$, we choose the one with the largest likelihood value to be the parameter, $\widetilde{\boldsymbol{\zeta}}$, for Warp-U bridge sampling. Simulations show that $M$ as small as 2 to 10 is sufficient to obtain a local maxima that serves well for the purpose of ensuring adequate overlap between $p$ and $\phi_{\text {mix }}$.

\subsection{Overcoming Adaptive Bias and Setting Tuning Parameters}

Let $\widetilde{\boldsymbol{\zeta}}_{\mathcal{D}}$ be the estimate of $\boldsymbol{\zeta}$ obtained by applying EM to all the draws from $p, \mathcal{D}=\left\{w_{1}, \ldots, w_{n}\right\}$, and let $\hat{\lambda}_{\mathcal{D}}^{(\mathrm{U})}=\log \left(\hat{c}_{\mathcal{D}}^{(\mathrm{U})}\right)$ be the corresponding Warp-U bridge sampling estimator. Because $\widetilde{\boldsymbol{\zeta}}_{\mathcal{D}}$ is a function of the draws from $p$, the distribution of the corresponding Warp- $\mathrm{U}$ transformed 


\begin{tabular}{|c|c|ccc}
\hline $\mathrm{EM}$ & & $\mathrm{BS}$ & $\rightarrow$ & $\hat{\lambda}_{\mathrm{H}_{2}}^{(\mathrm{U})}$ \\
& $\mathrm{BS}$ & $\mathrm{EM}$ & $\rightarrow$ & $\hat{\lambda}_{\mathrm{H}_{2}}^{(\mathrm{U})}$
\end{tabular}$\rightarrow \hat{\lambda}_{\mathrm{H}}^{(\mathrm{U})}=\frac{1}{2}\left(\hat{\lambda}_{\mathrm{H}_{1}}^{(\mathrm{U})}+\hat{\lambda}_{\mathrm{H}_{2}}^{(\mathrm{U})}\right)$

Figure 5: A strategy for removing the adaptive bias without (unduly) increasing the variance of the Warp-U bridge sampling estimator. Each $\hat{\lambda}_{\mathrm{H}_{i}}^{(\mathrm{U})}, i=1,2$ uses up to $50 \%$ of the draws from $p$ for estimating $\zeta$ and the other $50 \%$ for Warp-U bridge sampling. We then average the two estimators.

draws, $\left\{\tilde{w}_{1}, \ldots, \tilde{w}_{n}\right\}$, is no longer proportional to $\tilde{q}(\cdot ; \boldsymbol{\zeta})$ in $(17)$ when we substitute $\boldsymbol{\zeta}=\widetilde{\boldsymbol{\zeta}}_{\mathcal{D}}$. In other words, $\hat{\lambda}_{\mathcal{D}}^{(\mathrm{U})}$ has an adaptive bias induced by the dependence of $\widetilde{\boldsymbol{\zeta}}_{\mathcal{D}}$ on $\mathcal{D}$, demonstrated in Figure 6 (see Section 4.3).

Since the additional bias of $\hat{\lambda}_{\mathcal{D}}^{(\mathrm{U})}$ is due to the dependence of $\widetilde{\boldsymbol{\zeta}}_{\mathcal{D}}$ on the draws from $p$, an obvious remedy is to use two disjoint subsets of the draws from $p$ for estimating $\zeta$ and for bridge sampling. We can then switch the roles of these subsets to gain more statistical efficiency. Figure 5 depicts the sub-sampling strategy we use to obtain two separate bridge sampling estimators, $\hat{\lambda}_{\mathrm{H}_{i}}^{(\mathrm{U})}, i=1,2$. Each $\hat{\lambda}_{\mathrm{H}_{i}}^{(\mathrm{U})}$ is obtained by using $L \leqslant n / 2$ of the draws from $p$ to estimate $\boldsymbol{\zeta}$ and the other $50 \%$ of the draws for the Warp-U bridge sampling specified by the estimated $\boldsymbol{\zeta}$. Our final estimator $\hat{\lambda}_{\mathrm{H}}^{(\mathrm{U})}$ is the average of $\hat{\lambda}_{\mathrm{H}_{1}}^{(\mathrm{U})}$ and $\hat{\lambda}_{\mathrm{H}_{2}}^{(\mathrm{U})}$. Our empirical investigations, under the setting of i.i.d. draws from $p$ and $\phi$, suggest that the correlation between $\hat{\lambda}_{\mathrm{H}_{1}}^{(\mathrm{U})}$ and $\hat{\lambda}_{\mathrm{H}_{2}}^{(\mathrm{U})}$ is often very small (e.g., $<0.06$ ). Thus, when the i.i.d. assumption approximately holds, the variance of $\hat{\lambda}_{\mathrm{H}}^{(\mathrm{U})}$ is nearly half that of $\hat{\lambda}_{\mathrm{H}_{i}}^{(\mathrm{U})}$.

As depicted in Figure 5, we may choose $L<n / 2$ in order to reduce the EM computation, which is a reasonable strategy given that $\phi_{\text {mix }}$ does not need to be a very precise approximation to $p$. Appendix B provides further practical guidance for setting $K, L$, and $m$ (the number of draws from $\phi$ ), which we now summarize. Firstly, we suggest setting $K \leq n / 100$ because our simulations suggest that $\phi_{\text {mix }}$ tends to overfit for $K>n / 100$ which can even cause the RMSE of $\hat{\lambda}_{\mathrm{H}}^{(\mathrm{U})}$ to increase. Another reason to avoid large $K$ is that computational cost increases quadratically with $K$. Next, we found that a reasonable choice of $L$ is $\min (50 K, n / 2)$ because the reductions in the RMSE of $\hat{\lambda}_{\mathrm{H}}^{(\mathrm{U})}$ are relatively small when we increase $L$ past $50 K$. Lastly, in terms of precision per CPU second, when $K$ is already large, increasing $m$ is a more efficient strategy for reducing the variance of $\hat{\lambda}_{\mathrm{H}}^{(\mathrm{U})}$ than increasing $K$ further. However, when $K$ is small it is often more efficient to increase $K$ rather than $m$, because if there are fewer components in $\phi_{\text {mix }}$ than there are major modes of $p$, or if the modes of $p$ are asymmetric or heavy tailed, then large reductions in RMSE can usually be obtained by increasing $K$.

\subsection{Examples in 10 and 50 dimensions}

To illustrate the effectiveness of using diagonal covariance matrices and the above bias reduction strategy, we first consider a 10 dimensional example where $p$ is set to be a mixture of 25 mul- 

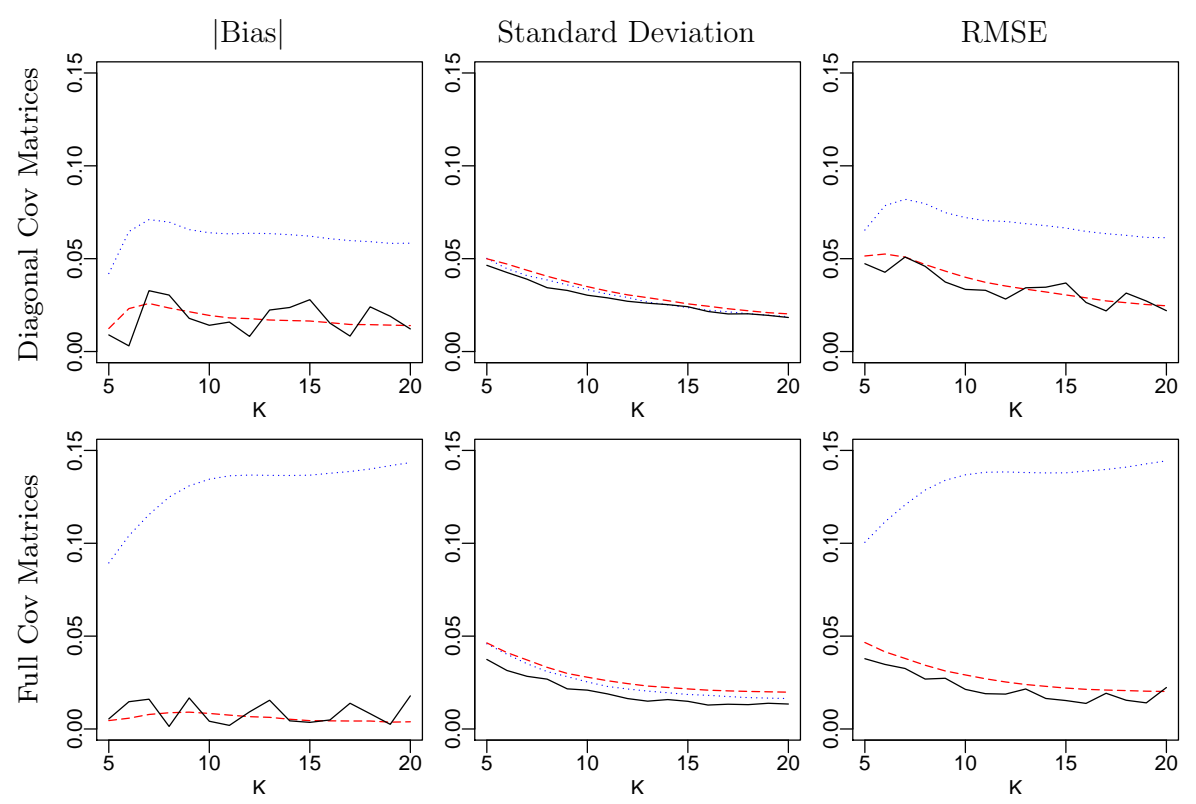

Figure 6: The columns show the $\mid$ bias $\mid$, the standard deviation, and the RMSE of (i) $\hat{\lambda}_{\mathcal{D}, \mathcal{Z}}^{(\mathrm{U})}=\log \left(\hat{c}_{\mathcal{D}, \mathcal{Z}}^{(\mathrm{U})}\right)$ (dotted lines), the Warp-U estimator specified by $\widetilde{\boldsymbol{\zeta}}_{\mathcal{D}}$, which is estimated from $\mathcal{D}=\left\{w_{1}, \ldots, w_{n}\right\}$, (ii) $\hat{\lambda}_{\mathcal{I}, \mathcal{Z}}^{(\mathrm{U})}=\log \left(\hat{c}_{\mathcal{I}, \mathcal{Z}}^{(\mathrm{U})}\right)$ (solid lines), Warp-U specified by $\widetilde{\zeta}_{\mathcal{I}}$, which is independent of $\mathcal{D}$, and (iii) (dashed lines) the average of two Warp-U bridge sampling estimators with half of the draws from $p$ for estimating $\boldsymbol{\zeta}$ and the other half for bridge sampling. The subscript " $\mathcal{Z}$ " indicates "Diag" (top row) or "Full" (bottom row) covariance matrices in the Gaussian mixture model.

tivariate skew- $t$ distributions, whose density is given in the R package 'sn' by Azzalini (2011), also see Azzalini (2013). We specify the degrees of freedom of the 25 skew- $t$ distributions to take various values between 1 and 4, the skew parameters to take values between -100 and 200, and the scale matrices to be non-sparse. To evaluate our methods properly, we simulate $10^{4}$ replicate data sets, each of which contains 2500 independent draws from $p$.

We consider three Warp- $\mathrm{U}$ bridge sampling estimators, with diagonal covariance matrices for $\phi_{\text {mix }}$. They are $\hat{\lambda}_{\mathcal{D}, \mathcal{D} \text { iag }}^{(U)}, \hat{\lambda}_{\mathrm{H}, \mathcal{D} \text { iag }}^{(\mathrm{d})}$, and $\hat{\lambda}_{\mathrm{I}, \mathcal{D} \text { iag }}^{(\mathrm{U})}$, where the first subscript specifies whether $\hat{\lambda}$ is computed by estimating $\zeta$ using all the draws from $p(\mathcal{D})$, by setting $L=n / 2$ and using the scheme in Section $4.2(\mathrm{H})$, or by estimating $\boldsymbol{\zeta}$ from an independent set of draws $(\mathcal{I})$. For all three estimators we use the optimal choice of $\alpha$ and set $m=2500$, i.e., the number of independent draws from the auxiliary $\phi=\mathcal{N}\left(0, I_{10}\right)$. The estimator $\hat{\lambda}_{\mathcal{I}, \text { Diag }}^{(\mathrm{U})}$ serves as a benchmark for comparison because it is free of adaptive bias.

The lines in the top row of Figure 6 show the bias (left panel), the standard deviation (center panel), and the RMSE (right panel) of the three estimators: $\hat{\lambda}_{\mathcal{D}, \mathcal{D i a g}}^{(\mathrm{U})}$ (dotted lines), $\hat{\lambda}_{\mathrm{H}, \mathcal{D} \text { iag }}^{(\mathrm{U})}$ (dashed lines), and $\hat{\lambda}_{\mathrm{I}, \mathcal{D}_{\text {iag }}^{(\mathrm{U}}}^{\mathrm{R}}$ (solid lines). Results are plotted for all values of $K$ between 5 and 20 inclusive. Larger values of $K$ generally represent a better approximation to $p$ but more computation (and potentially less gain from using Warp-U bridge sampling as opposed to standard bridge sampling 

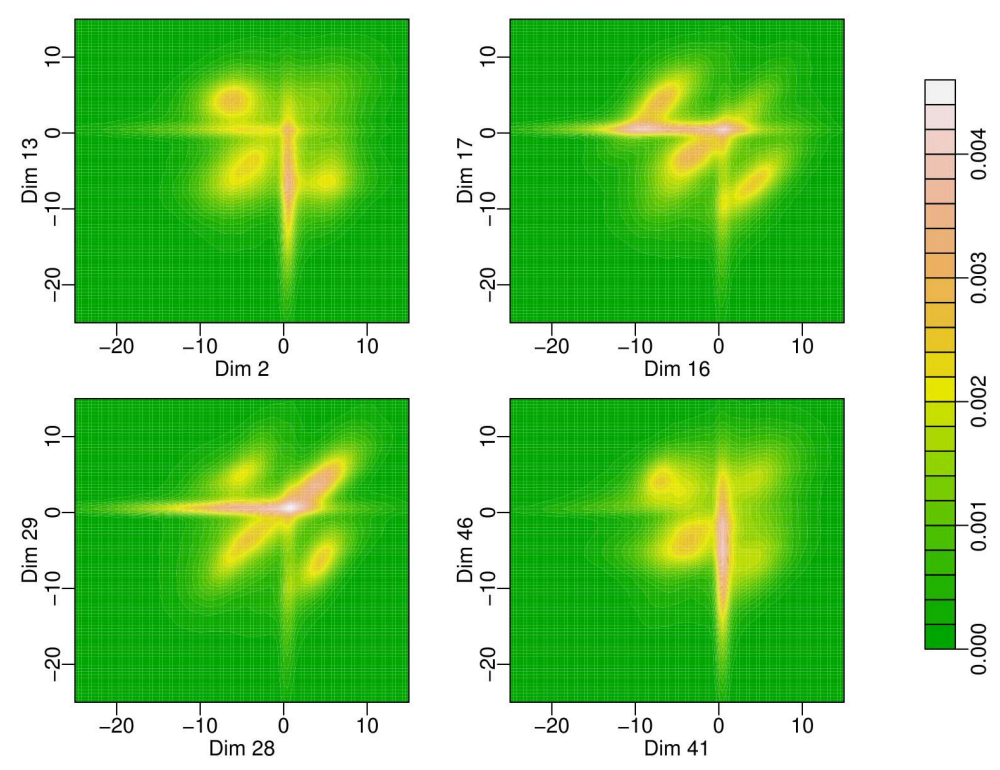

Figure 7: Contours of the density $p$ projected onto different pairs of dimensions.

between $p$ and $\left.\phi_{\text {mix }}\right)$. The top left panel of Figure 6 shows the excessive bias of $\hat{\lambda}_{\mathcal{D}, \mathcal{D} \text { iag }}^{(\mathrm{U})}$ compared with $\hat{\lambda}_{\mathcal{I}, \mathcal{D} \text { iag }}^{(\mathrm{U})}$. In contrast, the bias of our bias adjusted estimator, $\hat{\lambda}_{\mathrm{H}, \mathcal{D} \text { iag }}^{(\mathrm{U})}$, is as low as that of the benchmark $\hat{\lambda}_{\mathcal{I}, \mathcal{D i a g}}^{(\mathrm{u})}$. In the top center panel of Figure 6 we see that the variances of all three estimators are very similar, and decrease as $K$ increases. The decrease is because on average larger $K$ corresponds to more overlap between $p$ and the calibrated $\phi_{\text {mix }}$, and thus more overlap between $\tilde{p}$ and $\phi$. The top right panel of Figure 6 shows the RMSE of the estimators which is similar for $\hat{\lambda}_{\mathrm{H}, \mathcal{D} \text { iag }}^{(\mathrm{U})}$ and $\hat{\lambda}_{\mathcal{I}, \mathcal{D} \text { iag }}^{(\mathrm{U})}$, but much larger for $\hat{\lambda}_{\mathcal{D}, \mathcal{D} \text { iag }}^{(\mathrm{U})}$ because of its large bias.

The bottom row of Figure 6 shows similar results to those discussed above, but in the case where the covariance matrices of the components of $\phi_{\text {mix }}$ are not constrained to be diagonal. In this setting, we denote the three estimators by $\hat{\lambda}_{\mathcal{D}, \text { Full }}^{(\mathrm{U})}, \hat{\lambda}_{\mathrm{H}, \text { Full }}^{(\mathrm{U})}$, and $\hat{\lambda}_{\mathrm{I}, \mathrm{Full}}^{(\mathrm{U})}$. The results broadly match those in the top row of Figure 6, except that the bias (bottom left panel) and RMSE (bottom right panel) of $\hat{\lambda}_{\mathcal{D}, \text { Full }}^{(\mathrm{U})}$ are even larger than those of $\hat{\lambda}_{\mathcal{D}, \mathcal{D} \text { iag }}^{(\mathrm{U})}$. This is because with full covariance matrices, we have significantly more parameters to be estimated, and hence more substantial over-fitting bias. However, Figure 6 clearly shows that our method removes the adaptive bias regardless of its magnitude, and differences between using full and diagonal covariance matrices when fitting $\phi_{\text {mix }}$ are minor (compare the dashed lines in the top and bottom panels). Since fitting $\phi_{\text {mix }}$ with diagonal covariance matrices is computationally much less expensive, $\hat{\lambda}_{\mathrm{H}, \mathcal{D} \text { iag }}^{(\mathrm{U})}$ achieves better RMSE per CPU second than $\hat{\lambda}_{\mathrm{H}, \mathrm{Full}}^{(\mathrm{U})}$; see Appendix C for further demonstration. Hence, from hereon we always use diagonal covariance matrices and the estimation strategy in Section 4.2 and denote the final estimator by $\hat{\lambda}_{\alpha}^{(\mathcal{X})}=\frac{1}{2}\left(\hat{\lambda}_{\alpha, 1}^{(\mathcal{X})}+\hat{\lambda}_{\alpha, 2}^{(\mathcal{X})}\right)$, where $\mathcal{X}=U$ or "mix". Recall that "mix" refers to the ordinary bridge sampling estimator using $p$ 

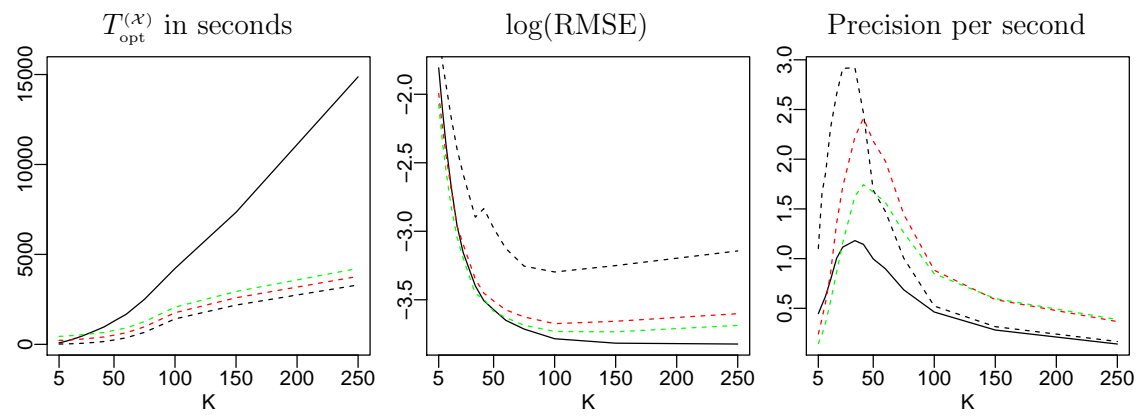

Figure 8: The total computational cost (left), the $\log (\mathrm{RMSE})$ (middle), and the $P p S$ (right) of $\hat{\lambda}_{\mathrm{opt}}^{(\mathrm{U})}$ (solid lines, $m=n$ ) and $\hat{\lambda}_{\text {opt }}^{\text {(mix) }}$ (dashed lines) with $m=n$ (black), $16 n$ (red), and $32 n$ (green).

and $\phi_{\text {mix }}$, i.e., the logarithm of (19).

Next we consider a 50 dimensional example. For this example, $p$ is a mixture of 30 distributions, including Normal distributions, $t$-distributions (including Cauchy distributions), and multivariate distributions with gamma and/or exponential marginal distributions and Normal copulas. The four 2-D projection contour plots of $p$ in Figure 7 show the density has very long tails and is quite skewed in some directions. Evaluating $p$ is about 700 times more costly than evaluating $\phi$ (the auxiliary density). The simulation results are based on $10^{4}$ replications, and in each replication, $n=10^{4}$ samples were drawn from $p$.

Figure 8 shows the total computational cost, the RMSE, and the PpS ("precision per second", defined as the reciprocal of RMSE $\times$ CPU seconds) of $\hat{\lambda}_{\mathrm{opt}}^{(\mathcal{X})}$. As in the 10-dimensional example, the RMSE decreases as $K$ increases up to $n / 100$, and when $K>n / 100$, the mixture model overfits the data (i.e., the draws from $p$ ), resulting in a slight increase in the RMSE of $\hat{\lambda}_{\text {opt }}^{\text {(mix) }}$. On average, $\log (\mathrm{RMSE})$ of $\hat{\lambda}_{\mathrm{opt}}^{(\mathrm{U})}$ is about $60 \%$ of that of $\hat{\lambda}_{\mathrm{opt}}^{(\mathrm{mix})}$, but the computational cost of $\hat{\lambda}_{\mathrm{opt}}^{(\mathrm{U})}$ is 4.7 times that of $T_{\mathrm{opt}}^{(\mathrm{mix})}$, so in terms of the $P p S, \hat{\lambda}_{\mathrm{opt}}^{(\mathrm{mix})}$ is superior to $\hat{\lambda}_{\mathrm{opt}}^{(\mathrm{U})}$. In addition, for large $K$, when we increase $m$ from $n$ (black lines) to $16 n$ (red) and $32 n$ (green), the total computational cost of $\hat{\lambda}_{\mathrm{opt}}^{(\mathrm{mix})}$ increases only by a small fraction, but the gain in statistical efficiency is substantial. Thus, in this illustration $\hat{\lambda}_{\mathrm{opt}}^{(\mathrm{mix})}$ is preferred to $\hat{\lambda}_{\mathrm{opt}}^{(\mathrm{U})}$. However, such preferences vary with machines and implementations, because we have not optimized the function evaluation routines or other aspects of the code. Furthermore, the relatively high computational cost of $\hat{\lambda}_{\mathrm{opt}}^{(\mathrm{U})}$ seen in Figure 8 is partly due to obtaining the Warp-U transformed draws $\left\{\tilde{\omega}_{1}, \ldots, \tilde{\omega}_{n}\right\}$, which does not require any target evaluations. In other scenarios, evaluations of $q$ may dominate the computational cost more, and then computational efficiency would depend mostly on the number of target evaluations. In particular, in such cases, increasing $m$ from $n$ to say $16 n$ would represent an 8.5 (i.e, $(16+1) / 2)$ ) multiplicative increase in computation, as opposed to the relatively modest increase seen in the left panel of Figure 8, and therefore the computational cost of $\hat{\lambda}_{\mathrm{opt}}^{(\mathrm{mix})}$ would be more similar to that of $\hat{\lambda}_{\mathrm{opt}}^{(\mathrm{U})}$ for a given $\log (\mathrm{RMSE})$. We consider measuring computational 
efficiency by the number of target evaluations in Section 5 , and find $\hat{\lambda}_{\mathrm{opt}}^{(\mathrm{mix})}$ and $\hat{\lambda}_{\mathrm{opt}}^{(\mathrm{U})}$ to be closely comparable. Opportunities for reducing the computational cost associated with Warp-U bridge sampling will be discussed in Section 6 .

\section{Warp-U extension of Generalized Wang-Landau method}

As we emphasized earlier, bridge sampling is applicable to general MCMC settings. We therefore do not need the draws to be independent, as long as they are from the target $p$, or at least in the long run. There are cases, however, where the available draws are from a distribution that is known to be different from the target $p$. Indeed, some of the most promising approaches for estimating normalizing constants combine the tasks of sampling and estimation into one coherent algorithm, but often do not directly sample from $p$. An important case of such a combined approach is the generalized Wang-Landau (GWL) algorithm proposed by Liang (2005). GWL is particularly useful in the current context because of its ability to efficiently sample from highly multi-modal distributions. We therefore take it as a benchmark, and illustrate how it can be combined with Warp-U bridging sampling to obtain an improved estimator for normalizing constants. More generally, our strategy of incorporating a Warp-U bridge sampling step can be tried on other algorithms that combine sampling and estimation of normalizing constants.

\subsection{GWL algorithm}

We begin by briefly describing GWL, which shares some similarities with other energy based methods such as the equi-energy sampler (Kou et al., 2006). Suppose that we want to compute the integral $\int_{S} q(\omega) \mathbf{u}(\mathrm{d} \omega)$, for some unnormalized density $q$ and bounded region $S$. Typically $S$ is the region over which $q$ has non-negligible density, i.e., $q(S) \approx 1$. We divide $S$ into $r$ subregions

$S_{1}, \ldots, S_{r}$, which are defined by target energy bins; that is, within each $S_{i}$, the energy level, defined by $-\log q$, is roughly the same. Let the current estimate of the integral $\int_{S_{i}} q(\omega) \mathbf{u}(\mathrm{d} \omega)$ be denoted by $\hat{g}\left(S_{i}\right)$, and set the initial estimate to be $\hat{g}\left(S_{i}\right)=1$, for $i=1, \ldots, r$. GWL takes the inputs $n_{0}, \delta_{0}$, and $T$ (e.g., $n_{0}=1000, \delta_{0}=e-1 \approx 1.718, T=25$ ) and proceeds as detailed below. Note that, Liang (2005) introduces an additional tuning parameter, which is not needed when the goal is to estimate normalizing constants, and therefore it is not included here.

\section{GWL algorithm.}

For stage $t=1, \ldots, T$ :

1. Set $n_{t}=n_{t-1}(1.1)^{t-1}, \delta_{t}=\sqrt{1+\delta_{t-1}}-1$, and $\hat{g}\left(S_{i}\right)^{(t, 1)}=\hat{g}\left(S_{i}\right)^{\left(t-1, n_{t-1}\right)}$, for $i=1, \ldots, r$. 2. For $k=1, \ldots, n_{t}$ do the following:

(i) Use a Metropolis-Hastings step, with proposal density $h$, to draw a sample $\omega$ from the current target density 


$$
\psi^{(t, k)}(\omega) \propto \sum_{i=1}^{r} \frac{q(\omega)}{\hat{g}^{(t, k)}\left(S_{i}\right)} I\left(\omega \in S_{i}\right) .
$$

The $(t, k)$ superscripts indicate that the current target and the estimate of $g\left(S_{i}\right)$, for $i=1, \ldots, r$, are updated in each iteration within each stage.

(ii) Update $\hat{g}^{(t, k)}\left(S_{I_{\omega}}\right)$ to $\left(1+\delta_{t}\right) \hat{g}^{(t, k)}\left(S_{I_{\omega}}\right)$, where $I_{\omega}$ is the index such that $\omega \in S_{I_{\omega}}$, i.e., a regional mass estimate $\hat{g}^{(t, k)}\left(S_{i}\right)$ is increased only if $S_{i}$ contains the draw $\omega$.

It should be clear from the description above that, in the limit, GWL samples the subregions $S_{1}, \ldots, S_{r}$ with equal probability, and within each $S_{i}$, it samples according to $q$. Therefore its stationary distribution is not the targeted $q$, but what can intuitively be described as a "re-distributed" $q$ that equalizes the masses of the energy bins:

$$
\psi(\omega)=\sum_{i=1}^{r} \frac{q(\omega)}{g\left(S_{i}\right)} I\left(\omega \in S_{i}\right)
$$

Liang (2005) verified this convergence assuming that the intermediate densities of (20) can be sampled from exactly. Practically, we can sample from them approximately, say by repeating the Metropolis-Hastings step many times between each update of the estimate of $g\left(S_{i}\right)$, for $i=1, \ldots, r$. However, Liang (2005) used only one Metropolis-Hastings update in his illustrations, and we follow this practice (but with an ideal proposal, see Appendix D). His proof also assumed $n_{t}$ grows sufficiently fast with $t$, but the multiplicative factor 1.1 suggested in Liang (2005) may not always be adequately large, though it seems computationally problematic to increase it much further.

Since GWL samples the subregions uniformly, the final estimators $\hat{g}\left(S_{i}\right)=\hat{g}^{\left(T, n_{T}\right)}\left(S_{i}\right)$ estimate the integrals $g\left(S_{i}\right)$ only up to a common constant, denoted $A$, which depends on $\delta_{0}$ and the number of iterations made at each stage of the algorithm. To estimate the log normalizing constant of $q$, namely $\lambda=\log (c)$, we must remove $A$, which can be done by running GWL with a modified version of $q$ as we now explain. Choose $S_{2}, \ldots, S_{r}$ to be such that $\left(\bigcup_{i=2}^{r} S_{i}\right)^{c}$ has negligible mass under $q$, and choose $S_{1} \subset\left(\bigcup_{i=2}^{r} S_{i}\right)^{c}$ such that its volume $\left|S_{1}\right|$ is finite and known (Liang, 2005). Next, run GWL with $q$ replaced by

$$
q_{\text {mod }}(\omega)=\left\{\begin{array}{cc}
q(\omega) & \text { for } \omega \in \bigcup_{i=2}^{r} S_{i} \\
\frac{1}{\left|S_{1}\right|} & \text { for } \omega \in S_{1} \\
0 & \text { otherwise. }
\end{array}\right.
$$

The basic idea is that on $S_{1}$ we can treat $q$ as uniform since its actual distribution contributes little to the normalizing constant of $q$. Lastly, for each $S \in\left\{S_{2}, \ldots, S_{r}\right\}$, Liang (2005) estimated the integral $\int_{S} q(\omega) d \omega$ by $\hat{g}(S) / \hat{g}\left(S_{1}\right)$. Assuming convergence of GWL, a consistent estimate of $\lambda$ is thus given by $\hat{\lambda}_{G W L}=\log \left(\sum_{i=2}^{r} \hat{g}\left(S_{i}\right) / \hat{g}\left(S_{1}\right)\right)$.

The key strengths of GWL are its adaptive nature and that it exhibits good mixing properties even for multi-modal targets, the latter property being a benefit of asymptotic uniform sampling 
across energy bins. In practice, a limitation of the algorithm is that the MSE of $\hat{\lambda}_{G W L}$ is bounded below for fixed $n_{0}$ and the specified geometric growth in $n_{t}$, as can be seen in the top left panel of Figure 9 (discussed in Section 5.3). (Liang et al. (2007) attempted to mitigate this phenomenon, but the convergence properties of the updated algorithm again may not be ideal, and further developments are still being made; see for example Jacob and Ryder (2014).) Here we simply view GWL as a related method to compare against and combine with. For these purposes the lower bound on the convergence of $\hat{\lambda}_{G W L}$ does not play a large role because the number of target evaluations we allow is approximately equal to or lower than the number required by GWL to achieve its minimum MSE. For further details of GWL the reader is referred to Liang (2005), Liang et al. (2007), Bornn et al. (2013), and Jacob and Ryder (2014).

\subsection{Combining GWL with Warp-U}

Consider a situation where GWL has been run for $T^{*}<T$ stages. We suspect that it is near convergence, and want to determine if we can reduce the MSE by using the computation to only complete the remaining $T-T^{*}$ stages (the GWL-only approach) or to make the use of Warp-U bridge sampling to obtain the estimator $\hat{\lambda}_{\mathrm{opt}}^{(\mathrm{U})}$ (the GWL+Warp approach). Draws from our target $p$ are required to implement the latter approach, but can be obtained from the GWL run without any additional target evaluations. To see this, first let $G_{i}$ denote the set of samples collected from subregion $S_{i}$ during the $T^{*}$ stages of the GWL run, for $i=1, \ldots, r$. With this notation, we propose the following addition to GWL.

\section{Warp-U Addition.}

1. For $l=1, \ldots, n$, repeat the following two steps:

(i) Sample a subregion index $k \in\{2, \ldots, r\}$ using the probabilities $b_{i} \propto \hat{g}\left(S_{i}\right) 1_{\left\{G_{i} \neq \emptyset\right\}}$, for $i=2, \ldots, r$.

(ii) With uniform sampling, select a sample $\omega_{l} \in G_{k}$, i.e., select one of the samples in subregion $S_{k}$ collected by GWL.

2. Apply Warp-U bridge sampling with $\left\{\omega_{1}, \ldots, \omega_{n}\right\}$ and $m$ draws from $\phi$ to obtain the estimate $\hat{\lambda}_{\mathrm{opt}}^{(\mathrm{U})}$.

The first step obtains draws from $p$ restricted to $\bigcup_{i=2}^{r} S_{i}$, and the second step applies Warp-U bridge sampling using these draws. The indicator $1_{\left\{G_{i} \neq \emptyset\right\}}$ in $b_{i}$ indicates that we sample only regions from which there are samples during the GWL run. Since $q\left(\omega_{l}\right)$, for $l=1, \ldots, n$, has already been evaluated during the GWL run, the only new target evaluations required for the above Warp-U addition are the $m K$ needed to evaluate $\tilde{q}$ at the $m$ draws from $\phi$. In step 2 above, we could alternatively apply standard bridge sampling to $p$ and $\phi_{\text {mix }}$ to obtain $\hat{\lambda}_{\mathrm{opt}}^{\text {mix }}$, as described in Section 3.3. We refer to this alternative approach as GWL+BS. 

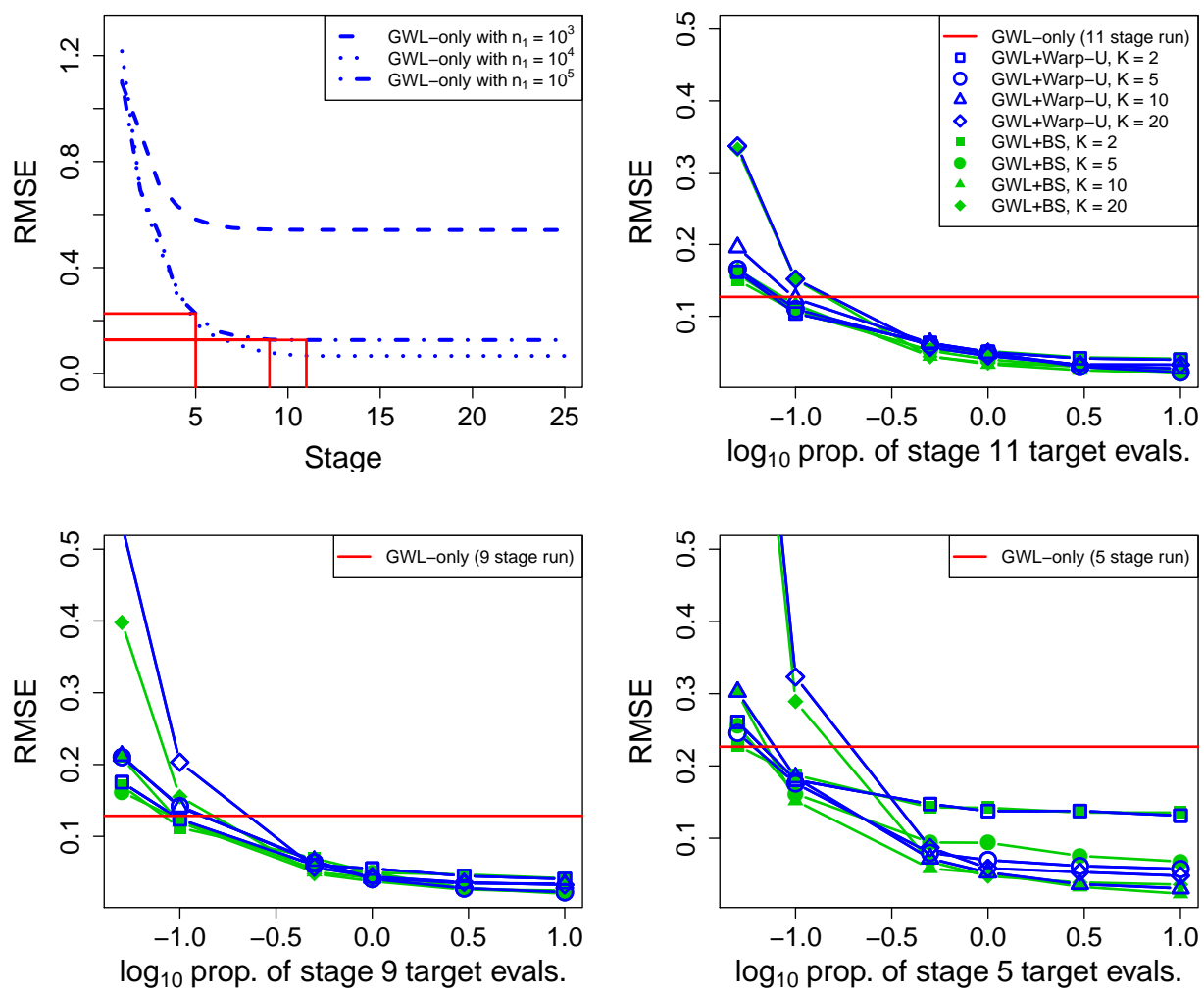

Figure 9: The top left panel shows the RMSE of $\hat{\lambda}_{G W L}$ at each of 25 stages, for $n_{0}=10^{3}, 10^{4}, 10^{5}$. The solid lines indicate the results for 11-stage, 9-stage, and 5-stage runs of the GWL-only algorithm with $n_{0}=10^{4}$. The top right panel shows the RMSE of the GWL+Warp-U estimator (hollow symbols) and the GWL+BS estimator (solid symbols), where the initial GWL run is for 10 stages. The number of target evaluations used by the Warp- $\mathrm{U}$ or ordinary bridge sampling step is given on the $x$-axis as a proportion of the target evaluations that would be needed for an eleventh stage of GWL (on a $\log _{10}$ scale). The different shapes correspond to different settings of $K$. The bottom left and right panels show similar results where the initial GWL run is for 8 and 4 stages, respectively.

\subsection{Illustration of the GWL+Warp-U algorithm}

We consider the 25 skewed- $t$ mixture example from Section 4.2. We run the GWL-only algorithm for $T=25$ stages, and again set $\delta_{0}=e-1$. We try $n_{0}=10^{3}, 10^{4}, 10^{5}$ and find that larger $n_{0}$ requires more stages for the estimator $\hat{\lambda}_{G W L}$ to converge but leads to lower RMSE; see the top left panel of Figure 9. We choose $n_{0}=10^{4}$ as a compromise between RMSE and computational cost. The value of $n_{0}$ is not the target of our comparisons, nor is the choice of the proposal density $h$ or the partition $S_{1}, \ldots, S_{r}$. We therefore use our knowledge of the true target $p$ to configure these components to favor the GWL-only algorithm; see Appendix D for details.

The solid lines in the top left panel of Figure 9 indicate the RMSE when the GWL-only 
algorithm (with $n=10^{4}$ ) is run for 5, 9, and 11 stages, and we see the RMSE is stabilized after stage 9. For reference, the same three RMSE values are indicated by a horizontal line in the top right, bottom left, and bottom right panels of Figure 9, respectively. Running GWL for more than 9 stages does not improve the RMSE, but we can improve it using the Warp-U Addition detailed in Section 5.2, even if we do not increase the overall computational cost. In the top right panel of Figure 9, the hollow symbols show the RMSE for the GWL+Warp-U estimator $\hat{\lambda}_{\text {opt }}^{(\mathrm{U})}$, in the case where the GWL component was run for only 10 stages, thereby saving the computation needed for an $11^{\text {th }}$ stage to be used for Warp-U sampling. Of course, we may decide we do not need to use all of the saved computation. The $x$-axis of Figure 9 gives the number of target evaluations we use for the Warp-U step, in units of $\log _{10}$ of the proportion of target evaluations needed for an $11^{\text {th }}$ stage of GWL, i.e., at 0 on the $x$-axis the computational cost of the Warp-U sampling matches that of an $11^{\text {th }}$ stage. The different hollow shapes correspond to different values of $K$ (number of mixture components). For comparison, the horizontal line indicates the RMSE under the GWL-only algorithm after 11 stages (for which the $x$-axis is irrelevant). At 0 on the $x$-axis the GWL-only and GWL+Warp- $\mathrm{U}$ methods use the same number of target evaluations, but the GWL+Warp-U method yields substantially lower RMSE for all four values of $K(2,5,10$, and 20). Indeed, the RMSE obtained is even lower than that achieved by the GWL-only algorithm with $n_{0}=10^{5}$ after 11 (or 25) stages, which uses a factor of 10 more target evaluations than used by the GWL+Warp-U runs (with $n_{0}=10^{4}$ ). This example suggests that applying Warp- $\mathrm{U}$ when GWL is at or close to convergence offers a way to substantially lower RMSE with only the number of target evaluations required to run one more stage of GWL (or even fewer). The improvements offered by GWL+Warp-U are thus almost free because we can stop GWL one stage early, and in any case the number of target evaluations required by the Warp-U part is relatively low.

For further comparison, the solid shapes in the top right panel of Figure 9 show results for the GWL+BS method, i.e, where the two bridge sampling densities are $p$ and $\phi_{\text {mix }}$. Note that, at any given point on the $x$-axis the number of target evaluations is the same for the GWL+BS and GWL+Warp-U algorithms, which is achieved by setting $m=n(2 K-1)$ in the GWL+BS algorithm and $m=n$ in the GWL+Warp-U algorithm. We see that GWL+BS again achieves substantially lower RMSE than is obtained by simply running an $11^{\text {th }}$ stage of the GWL-only algorithm. The RMSE under the GWL+BS method is also seen to be marginally lower than that under the GWL+Warp- $\mathrm{U}$ method, but now that we have controlled the number of target evaluations, we see the performance is very similar.

Naturally, Warp-U or standard bridge sampling can be applied after any number of GWL stages; we do not have to wait until we are sure of convergence. To investigate this, we ran the GWL+Warp-U and GWL+BS algorithms again but where the initial GWL run was shorter. The bottom left and bottom right panel of Figure 9 show results in the case where the initial GWL 
run was 8 and 4 stages, respectively. These results are qualitatively similar to before, except that GWL+Warp-U occasionally performs slightly better than GWL+BS (e.g., when $K=5$ in the bottom right panel). The main constraint on when Warp-U or standard bridge sampling can be used is that GWL needs to have been run for long enough to have explored regions where $p$ has high density. Otherwise, weighted re-sampling of the GWL samples will not yield approximate draws from $p$. This is not a major issue in practice because, as can be seen in the top left panel of Figure 9, GWL substantially reduces RMSE in its initial stages, and it is likely to be the latter stages where applying Warp-U or standard bridge sampling is most appealing.

\section{$6 \quad$ Strategies for Making Further Improvements}

We have seen that stochastic Warp-U transformations can transform a multi-modal density $q$ into an approximate Normal density (or some other convenient auxiliary density $\phi$ ). The transformed density $\tilde{q}$ has the same normalizing constant $c$ as the original target density $q$. Furthermore, since the overlap between $\tilde{q}$ and the base density $\phi$ is typically large, performing bridge sampling with the transformed draws $\left\{\tilde{\omega}_{1}, \ldots, \tilde{\omega}_{n}\right\}$ produces an accurate estimate of $c$. In particular, Theorem 1 implies that the accuracy is equal to or greater than that obtained if we perform bridge sampling using the original draws $\left\{\omega_{1}, \ldots, \omega_{n}\right\}$ and the densities $q$ and $\phi_{\text {mix }}$. Thus, in terms of statistical efficiency, Warp- $U$ transformations are always beneficial, especially when the target density is multi-modal. However, there are still a number of important directions for further developments, especially regarding computational efficiency and identifying a good approximating mixture distribution $\phi_{\text {mix }}$, which we now discuss.

\subsection{Reducing the Cost of Transformed Density Evaluations}

From (17), it is seen that the density $\tilde{q}$ is at least $K$ times more expensive to evaluate than $q$. Consequently, in Figure 9 we saw that despite its greater statistical efficiency, Warp-U bridge sampling performs similarly to ordinary bridge sampling with $q$ and $\phi_{\text {mix }}$, after accounting for the number of target evaluations needed, i.e., evaluations of $q$. When evaluating $q$ is inexpensive, ordinary bridge sampling can perform substantially better than Warp-U bridge sampling, in terms of precision per CPU second, e.g., see Figure 8. The greater efficiency of ordinary bridge sampling in such cases is due to the many multivariate Normal evaluations required by Warp-U bridge sampling. These evaluations are not prohibitively expensive and in practice will often be of secondary concern to $q$ evaluations. Cases where $q$ is inexpensive to evaluate are generally of less interest because many methods will be practical in this scenario. Thus, we are primarily concerned with the statistical efficiency of Warp- $\mathrm{U}$ as a function of $q$ evaluations.

We therefore need to find ways to reduce the computational cost of evaluating $\tilde{q}$. Direct density approximation is unlikely to be fruitful in high dimensions. A plausible strategy is 
to randomly select only some of the $K$ terms in (17) to evaluate, as is done in the mixture sampling method of Elvira et al. (2019); they randomly partitioned the set of sampling densities and then used these partitions in computing importance weights. Specifically, the importance weight for a sample $\omega$ drawn from density $p_{s}$ is set to be the reciprocal of the average of the sampling densities in the same partition as $p_{s}$. We could apply a similar approach by viewing the terms $\left.c \tilde{p}^{(k)}(\tilde{\omega})=c p^{(k)}\left(S_{k} \tilde{\omega}+\mu_{k}\right)\right)$ in (17) as the weighted and unnormalized sampling densities. However, we do not know from which weighted component $c \tilde{p}^{(k)}$ each sample $\tilde{\omega} \sim \tilde{p}$ originated. Furthermore, the $c \tilde{p}^{(k)}$ incorporate unknown weights, so the unweighted mixture components of $\tilde{q}$ are not available and therefore we cannot weight them by their empirical weights, as is required by (generalized) bridge sampling, e.g., the weights $s_{i}$, for $i=1,2$, in (4). Regarding the unknown sampling component $c \tilde{p}^{(k)}$, it may be sufficient to stochastically impute the index of the "true" component by drawing from the conditional distribution $\varpi(\Psi \mid \omega)$ in (14). A possible solution to the second difficulty is to use the theoretical component weights incorporated in the $c \tilde{p}^{(k)}$ in the final bridge sampling estimator as opposed to the observed weights. In summary, there are feasible methods for improving the computational efficiency of Warp- $U$ bridge sampling, but the several potential strategies need to be further explored.

\subsection{Base and Mixture Distribution Selection}

So far we have focused on using the standard Normal as the base distribution $\phi$. But Theorem 1 implies that the $f$-divergence between $\left(\phi_{\text {mix }}, p\right)$ is larger than that between $(\phi, \widetilde{p})$ for any continuous densities $p$ and $\phi$. For heavy-tailed targets, using a $t$-distribution may be more effective for capturing the mass of $p$, that is, a $t$-density may allow us to use a mixture $\phi_{\text {mix }}$ with fewer components and thereby reduce computational costs. In other contexts, the support of $p$ may be bounded and then a base density with bounded support would be more appropriate. Perhaps in some scenarios it would make sense for $\phi_{\text {mix }}$ to be a mixture of several different base densities, in which case some modifications to the development here would be needed. With a different choice of the base function, a different method for fitting $\phi_{\text {mix }}$ would be required, e.g., for the $t$-distribution, we could use the approach of Peel and McLachlan (2000) to fit $\phi_{\text {mix }}$.

Secondly, although our approach for fitting the Warp-U parameters $\boldsymbol{\zeta}$ is promising in practice, it is almost certainly not optimal. Kong et al. (2003) showed that a standard bridge sampling estimator is in fact a maximum likelihood estimator (MLE), and it may be possible to use the same likelihood framework to find an optimal estimator for $\boldsymbol{\zeta}$. More specifically, let $\phi_{i}$ be the pdf

of $\mathcal{N}\left(\mu_{i}, \Sigma_{i}\right)$, for $i=1, \ldots, K$, and $\phi_{\text {mix }}=\sum_{i=1}^{K} \pi_{i} \phi_{i}$. Then the maximum likelihood estimator of $c$ (with $p=q / c$ as before) identified by Kong et al. (2003) is

$$
\hat{c}=\sum_{i=1}^{2} \sum_{j=1}^{n_{i}} \frac{q\left(w_{i, j}\right)}{n_{1} \hat{c}^{-1} q\left(w_{i, j}\right)+n_{2} \phi_{\text {mix }}\left(\omega_{i, j}\right)},
$$


where $\left\{\omega_{1,1}, \ldots, \omega_{1, n_{1}}\right\}$ are draws from $p$, and $\left\{\omega_{2,1}, \ldots, \omega_{2, n_{2}}\right\}$ are draws from $\phi_{\text {mix }}$. The estimator $(22)$ is the same as $\hat{c}_{\mathrm{opt}}^{(\mathrm{mix})}=\exp \left(\hat{\lambda}_{\mathrm{opt}}^{(\mathrm{mix})}\right)$. If Warp-U transformations can be correctly incorporated into this likelihood framework then we can use the MLE of $(c, \boldsymbol{\zeta})$ to improve upon our current approach. Whether using a maximum likelihood approach or not, it is especially important to investigate further how the number of components $K$ should be chosen.

\subsection{It's Time to Build a Bridge}

The vast majority of the MC literature is about improving MC sampling efficiency, that is, how to design MC sampling algorithms most effectively. In contrast, bridge sampling, with or without warp transformations, is about improving MC inference efficiency, that is, how to gain more precision with a given set of MC draws. Adding warp bridge sampling to GWL provides an example of bridging the sampling and analysis approaches, a strategy we believe has much more to offer than the current literature recognizes. We therefore invite interested readers to join us in laying further foundations for this much needed bridge.

\section{References}

Ali, S. M. and S. D. Silvey (1966). A general class of coefficients of divergence of one distribution from another. Journal of the Royal Statistical Society. Series B (Methodological) 28, 131-142.

Alspach, D. L. and H. W. Sorenson (1972). Nonlinear Bayesian estimation using Gaussian sum approximations. IEEE Transactions on Automatic Control 17(4), 439-448.

Azzalini, A. (2011). R package sn: The skew-normal and skew- $t$ distributions (version 0.4-17). URL http://azzalini.stat.unipd.it/SN.

Azzalini, A. (2013). The skew-normal and related families. Cambridge University Press, New York, NY.

Bennett, C. H. (1976). Efficient estimation of free energy differences from Monte Carlo data. Journal of Computational Physics 22(2), 245-268.

Berg, B. A. and T. Neuhaus (1991). Multicanonical algorithms for first order phase transitions. Physics Letters B 267(2), 249-253.

Bornkamp, B. (2011). Approximating probability densities by iterated Laplace approximations. Journal of Computational and Graphical Statistics 20(3), 656-669.

Bornn, L., P. E. Jacob, P. Del Moral, and A. Doucet (2013). An adaptive interacting WangLandau algorithm for automatic density exploration. Journal of Computational and Graphical Statistics 22(3), 749-773. 
Ceperley, D. M. (1995). Path integrals in the theory of condensed helium. Reviews of Modern Physics 67(2), 279-355.

Chen, J. and X. Tan (2009). Inference for multivariate normal mixtures. Journal of Multivariate Analysis 100(7), 1367-1383.

Chen, J., X. Tan, and R. Zhang (2008). Inference for normal mixtures in mean and variance. Statistica Sinica 18(2), 443-465.

Chib, S. (1995). Marginal likelihood from the Gibbs output. Journal of the American Statistical Association 90(432), 1313-1321.

Chib, S. and I. Jeliazkov (2001). Marginal likelihood from the Metropolis-Hastings output. Journal of the American Statistical Association 96(453), 270-281.

Day, N. E. (1969). Estimating the components of a mixture of normal distributions. Biometrika 56(3), 463-474.

DiCiccio, T. J., R. E. Kass, A. Raftery, and L. Wasserman (1997). Computing Bayes factors by combining simulation and asymptotic approximations. Journal of the American Statistical Association 92(439), 903-915.

Elvira, V., L. Martino, D. Luengo, and M. F. Bugallo (2015). Efficient multiple importance sampling estimators. IEEE Signal Processing Letters 22(10), 1757-1761.

Elvira, V., L. Martino, D. Luengo, M. F. Bugallo, et al. (2019). Generalized multiple importance sampling. Statistical Science 34(1), 129-155.

Gelman, A., J. B. Carlin, H. S. Stern, D. B. Dunson, A. Vehtari, and D. B. Rubin (2013). Bayesian data analysis. CRC Press, Boca Raton, FL.

Gelman, A. and X.-L. Meng (1998). Simulating normalizing constants: From importance sampling to bridge sampling to path sampling. Statistical Science 13, 163-185.

Gronau, Q. F., A. Sarafoglou, D. Matzke, A. Ly, U. Boehm, M. Marsman, D. S. Leslie, J. J. Forster, E.-J. Wagenmakers, and H. Steingroever (2017). A tutorial on bridge sampling. Journal of Mathematical Psychology 81, 80-97.

Gronau, Q. F., H. Singmann, and E.-J. Wagenmakers (2017). Bridgesampling: an R package for estimating normalizing constants. arXiv preprint arXiv:1710.08162.

Hesselbo, B. and R. B. Stinchcombe (1995). Monte Carlo simulation and global optimization without parameters. Physical Review Letters 74(12), 2151-2155. 
Hesterberg, T. (1995). Weighted average importance sampling and defensive mixture distributions. Technometrics 37(2), 185-194.

Jacob, P. E. and R. J. Ryder (2014). The Wang-Landau algorithm reaches the flat histogram criterion in finite time. The Annals of Applied Probability 24(1), 34-53.

Kass, R. E. and A. E. Raftery (1995). Bayes factors. Journal of the American Statistical Association 90(430), 773-795.

Kiefer, J. and J. Wolfowitz (1956). Consistency of the maximum likelihood estimator in the presence of infinitely many incidental parameters. The Annals of Mathematical Statistics 27, $887-906$.

Kong, A., P. McCullagh, X.-L. Meng, and D. Nicolae (2006). Further explorations of likelihood theory for Monte Carlo integration. In Advances in Statistical Modeling and Inference: Essays in Honor of Kjell A. Doksum (Ed: V. Nair), pp. 563-592. World Scientific Press.

Kong, A., P. McCullagh, X.-L. Meng, D. Nicolae, and Z. Tan (2003). A theory of statistical models for Monte Carlo integration (with discussions). Journal of the Royal Statistical Society: Series B (Statistical Methodology) 65(3), 585-604.

Kou, S., Q. Zhou, W. H. Wong, et al. (2006). Equi-energy sampler with applications in statistical inference and statistical mechanics. The Annals of Statistics 34(4), 1581-1619.

Liang, F. (2005). A generalized Wang-Landau algorithm for Monte Carlo computation. Journal of the American Statistical Association 100(472), 1311-1327.

Liang, F., C. Liu, and R. J. Carroll (2007). Stochastic approximation in Monte Carlo computation. Journal of the American Statistical Association 102(477), 305-320.

Liu, J. S., F. Liang, and W. H. Wong (2001). A theory for dynamic weighting in monte carlo computation. Journal of the American Statistical Association 96(454), 561-573.

Martino, L., V. Elvira, D. Luengo, and J. Corander (2017). Layered adaptive importance sampling. Statistics and Computing 27(3), 599-623.

Meng, X.-L. (2005). Comment: Computation, survey and inference. Statistical Science 20(1), 21-28.

Meng, X.-L. and S. Schilling (2002). Warp bridge sampling. Journal of Computational and Graphical Statistics $11(3), 552-586$.

Meng, X.-L. and W. H. Wong (1996). Simulating ratios of normalizing constants via a simple identity: A theoretical exploration. Statistica Sinica 6(4), 831-860. 
Mira, A. and G. Nicholls (2004). Bridge estimation of the probability density at a point. Statistica Sinica 14(2), 603-612.

Owen, A. and Y. Zhou (2000). Safe and effective importance sampling. Journal of the American Statistical Association 95(449), 135-143.

Peel, D. and G. J. McLachlan (2000). Robust mixture modelling using the $t$-distribution. Statistics and Computing 10(4), 339-348.

Romero, M. (2003). On two topics with no bridge: Bridge sampling with dependent draws and bias of the multiple imputation variance estimator. Ph. D. thesis, University of Chicago, Department of Statistics.

Shao, Q.-M. and J. G. Ibrahim (2000). Monte Carlo methods in Bayesian computation. Springer Series in Statistics, New York, NY.

Tan, Z. (2004). On a likelihood approach for Monte Carlo integration. Journal of the American Statistical Association 99(468), 1027-1036.

Tan, Z. (2013). Calibrated path sampling and stepwise bridge sampling. Journal of Statistical Planning and Inference 143(4), 675-690.

Veach, E. and L. J. Guibas (1995). Optimally combining sampling techniques for Monte Carlo rendering. In Proceedings of the 22nd Annual Conference on Computer Graphics and Interactive Techniques, pp. 419-428. ACM, New York, NY.

Villani, C. (2003). Topics in optimal transportation. Number 58. American Mathematical Society, Providence, RI.

Voter, A. F. (1985). A Monte Carlo method for determining free-energy differences and transition state theory rate constants. The Journal of Chemical Physics 82(4), 1890-1899.

Voter, A. F. and J. D. Doll (1985). Dynamical corrections to transition state theory for multistate systems: Surface self-diffusion in the rare-event regime. The Journal of Chemical Physics 82(1), 80-92.

Wang, F. and D. Landau (2001). Efficient, multiple-range random walk algorithm to calculate the density of states. Physical Review Letters 86(10), 2050-2053.

Wong, W. H. and F. Liang (1997). Dynamic weighting in monte carlo and optimization. Proceedings of the National Academy of Sciences 94(26), 14220-14224. 


\section{Disclaimer}

This document is being distributed for informational and educational purposes only and is not an offer to sell or the solicitation of an offer to buy any securities or other instruments. The information contained herein is not intended to provide, and should not be relied upon for, investment advice. The views expressed herein are not necessarily the views of Two Sigma Investments, LP or any of its affiliates (collectively, Two Sigma). Such views reflect the assumptions of the author(s) of the document and are subject to change without notice. The document may employ data derived from third-party sources. No representation is made by Two Sigma as to the accuracy of such information and the use of such information in no way implies an endorsement of the source of such information or its validity.

The copyrights and/or trademarks in some of the images, logos or other material used herein may be owned by entities other than Two Sigma. If so, such copyrights and/or trademarks are most likely owned by the entity that created the material and are used purely for identification and comment as fair use under international copyright and/or trademark laws. Use of such image, copyright or trademark does not imply any association with such organization (or endorsement of such organization) by Two Sigma, nor vice versa.

\section{Supplementary Material: Appendices}

\section{A Proof of Theorem 1}

Let $t(\theta, \omega)=\mathcal{F}_{\theta}(\omega)$, then we can write $\widetilde{W}=t(\Psi, W)$ and $\widetilde{X}=t(\Theta, X)$. Therefore, $\tilde{p}$ and $\phi$ are related to $p_{\Psi, W}$ and $\phi_{\Theta, X}$ of (15) respectively, via the same map $t: \Pi \times \Omega \rightarrow \Omega$. Claim (I) then follows from the monotone property of $f$-divergence (Ali and Silvey, 1966):

$$
\mathcal{D}_{f}(\tilde{p} \| \phi) \leqslant \mathcal{D}_{f}\left(p_{\Psi, W} \| \phi_{\Theta, X}\right)=\mathcal{D}_{f}\left(p \| \phi_{\text {mix }}\right),
$$

where the last equality holds because $p_{\Psi, W} / \phi_{\Theta, X}=p / \phi_{\text {mix }}$, a consequence of (15).

To prove (II), we use the fact from Ali and Silvey (1966) that, when $f$ is strictly convex, the inequality in (I) becomes an equality if and only if $t$ is a sufficient statistic for the distribution family $\left\{p_{\Psi, W}, \phi_{\Theta, X}\right\}$. By the well-known factorization theorem for sufficiency, the latter condition is the same as requiring that $p_{\Psi, W}(\theta, \omega) / \phi_{\Theta, X}(\theta, \omega)$ depends on $(\theta, \omega)$ only through $t=t(\theta, \omega)=$ $\mathcal{F}_{\theta}(\omega)$, almost surely with respect to $\mathbf{v} \times \mathbf{u}$. But from (15) and $\omega=\mathcal{F}_{\theta}^{-1}(t)=\mathcal{H}_{\theta}(t)$, we have

$$
p_{\Psi, W}(\theta, \omega) / \phi_{\Theta, X}(\theta, \omega)=p\left(\mathcal{H}_{\theta}(t)\right) / \phi_{\text {mix }}\left(\mathcal{H}_{\theta}(t)\right)=\ell(\theta ; t) .
$$

Consequently, $t$ is sufficient if and only if $\ell(\theta ; t)$ is free of $\theta$, and hence (II). 


\section{B Setting Tuning Parameters: Guidance from Simulation Studies}

We consider the tuning parameters for the estimator $\hat{\lambda}_{\mathrm{H}}^{(\mathcal{X})}=\frac{1}{2}\left(\hat{\lambda}_{\mathrm{H}_{1}}^{(\mathcal{X})}+\hat{\lambda}_{\mathrm{H}_{2}}^{(\mathcal{X})}\right)$ introduced in Section 4.2, where $\mathcal{X}=U$ or "mix". As in Section 4.3, we drop the subscript $\mathrm{H}$ (since we always use the estimation strategy in Section 4.2) and replace it by "opt" to indicate that here we use the optimal bridge $\alpha_{\mathrm{opt}}$ in (3). There are three tuning parameters:

- $K$ : the number of components in the normal mixture model $\phi_{\text {mix }}(\cdot ; \boldsymbol{\zeta})$;

- $L$ : the number of draws from $p$ for estimating $\boldsymbol{\zeta}$, with the restriction $L \leqslant n / 2$;

- $m$ : the number of draws from $N\left(0, I_{D}\right)$ or $\phi_{\text {mix }}$.

By investigating the performance of the estimators $\hat{\lambda}_{\mathrm{opt}}^{(\mathrm{U})}=\frac{1}{2}\left(\hat{\lambda}_{\mathrm{opt}, 1}^{(\mathrm{U})}+\hat{\lambda}_{\mathrm{opt}, 2}^{(\mathrm{U})}\right)$ and $\hat{\lambda}_{\mathrm{opt}}^{(\mathrm{mix})}=$ $\frac{1}{2}\left(\hat{\lambda}_{\text {opt }, 1}^{(\text {mix })}+\hat{\lambda}_{\text {opt }, 2}^{(\text {mix })}\right)$ under different choices of $(K, L, m)$, we gain practical guidance for choosing these tuning parameters. To form a sensible compromise between statistical and computational efficiency, we adopt precision per CPU second $(P p S)$, i.e., (Var $\times$ CPU seconds) ${ }^{-1}$ as our metric for evaluation. We set the sample size $n$ to be $10^{4}$, which is sufficiently large to allow us to investigate the impact of large $K$ on estimator performance. For each $(K, L, m)$, we generate $10^{4}$ replicate data sets, each consisting of $m$ independent draws from $p$, the 10-dimensional skewed- $t$ mixture density introduced in Section 4.2. If not specified, then $L=\min (50 K, n / 2)$ and $m=n$.

\section{B.1 Impact of $K$}

Figure 10 illustrates why the variance and the RMSE of $\hat{\lambda}_{\mathrm{opt}}^{(\mathrm{U})}$ in Figure 6 (Section 4) decrease as $K$ increases. The dotted line in Figure 10 is the scaled maximized $\log$-likelihood $\bar{l}_{\text {fit }}$, defined as

$$
\bar{l}_{\text {fit }}=\frac{1}{L} \sum_{i=1}^{L} \log \left(\phi_{\text {mix }}\left(w_{i} ; \tilde{\boldsymbol{\zeta}}_{L}\right)\right),
$$

where $\tilde{\boldsymbol{\zeta}}_{L}$ is the MLE of $\boldsymbol{\zeta}$ based on $\left\{w_{1}, \ldots, w_{L}\right\}$ and obtained via the EM algorithm. The quantity $\bar{l}_{\text {fit }}$ measures how well the calibrated $\phi_{\text {mix }}$ fits to the $L$ draws used for estimating $\boldsymbol{\zeta}$, and is an increasing function of $K$. The solid line in Figure 10 represents the scaled log-likelihood $\bar{l}^{*}$ for the other half of the draws from $p$, i.e.,

$$
\bar{l}^{*}=\frac{2}{n} \sum_{i=n / 2+1}^{n} \log \left(\phi_{\text {mix }}\left(w_{i} ; \tilde{\boldsymbol{\zeta}}_{L}\right)\right) .
$$

The quantity $\bar{l}^{*}$ provides an "out of sample" assessment of the fit of $\phi_{\text {mix }}$ to $p$. For moderate values of $K$, increases in $K$ (and hence increases in $\bar{l}_{\mathrm{fit}}$ ) generally result in increases in $\bar{l}^{*}$ because the mixture model better captures the mass of $p$. 
$l_{\text {fit }}$ and $l^{*}$

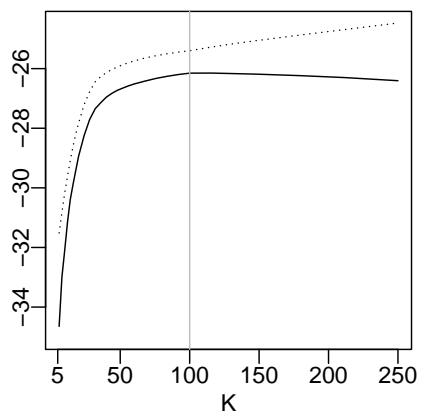

$l^{*}$ when $K \geqslant 100$

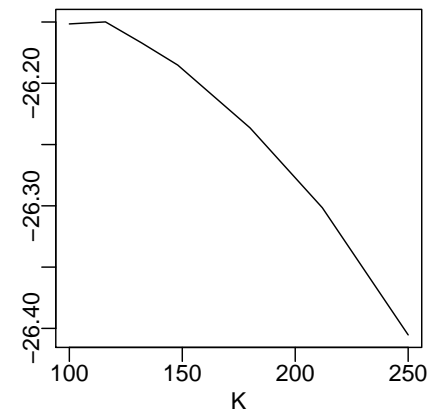

Figure 10: (Dotted line) $\bar{l}_{\mathrm{fit}}$; (solid lines) $\bar{l}^{*}$. The gray vertical line marks the value of $K$, around which $\bar{l}^{*}$ changes from increasing to decreasing as $K$ increases. The right-hand panel shows $\bar{l}^{*}$ for $K$ ranging from 100 to 250 (but notice the scale change on the vertical axis).
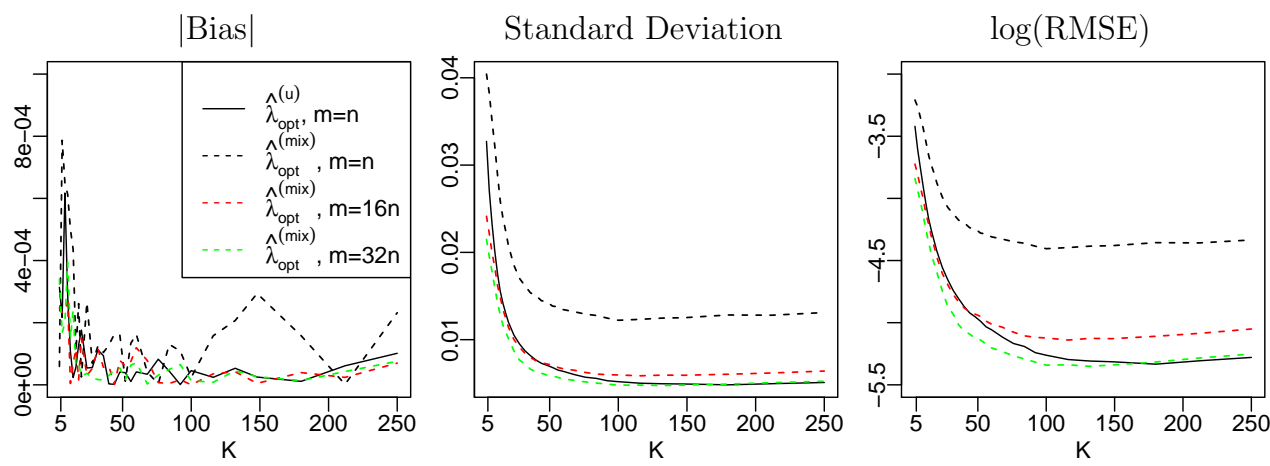

Figure 11: The three columns show |bias|, standard deviation and $\log (\mathrm{RMSE})$ of $\hat{\lambda}_{\mathrm{opt}}^{(\mathrm{U})}$ (solid lines) and $\hat{\lambda}_{\mathrm{opt}}^{\text {(mix) }}$ (dashed lines). Different colors correspond to different values of $m$ in the estimators. Black: $m=n$; Red: $m=16 n$; Green: $m=32 n$.

However, for a large $K$, the Normal mixture model overfits the $L \leqslant n / 2$ draws used to estimate $\boldsymbol{\zeta}$. Figure 10 (right) shows that $\bar{l}^{*}$ decreases slightly when $K$ exceeds 100 , indicating a slight increase in the divergence between $p$ and $\phi_{\text {mix }}\left(\cdot ; \tilde{\boldsymbol{\zeta}}_{L}\right)$. Figure 11 shows the $\mid$ bias $\mid$, standard deviation, and RMSE (on a logarithmic scale) of $\hat{\lambda}_{\mathrm{opt}}^{(\mathrm{U})}$ (solid lines) and $\hat{\lambda}_{\mathrm{opt}}^{\text {(mix) }}$ (dashed lines), for $K$ ranging from 5 to 250 . When $K$ exceeds $n / 100$, there is a slight increase in both the variance and the RMSE of these estimators as $K$ continues to increase. Thus, the statistical efficiency of the estimators suffers when overfitting occurs as expected.

Figure 12 (left) shows the computational cost of $\hat{\lambda}_{\text {opt }}^{(\mathrm{U})}$ (solid line) and $\hat{\lambda}_{\text {opt }}^{\text {(mix) }}$ (dashed line). When $K>n / 100$, the total number of CPU seconds $T_{\mathrm{opt}}^{(\mathrm{U})}$ exhibits quadratic growth as a function of $K$, whereas $T_{\mathrm{opt}}^{(\text {mix) }}$ grows linearly with $K$. Figure 12 (right) plots the $P p S$. The largest $P p S$ is obtained when $K$ is between 20 and 30 .

Based on the above simulations, we suggest setting $K \leqslant n / 100$ to avoid overfitting to the 
Median CPU seconds

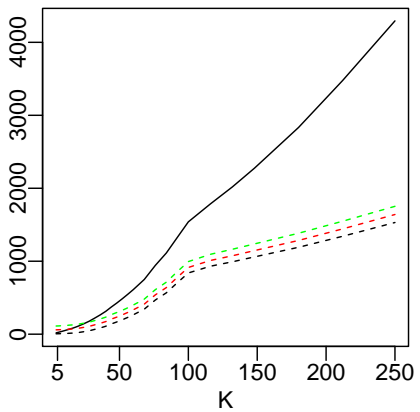

Precision per CPU second

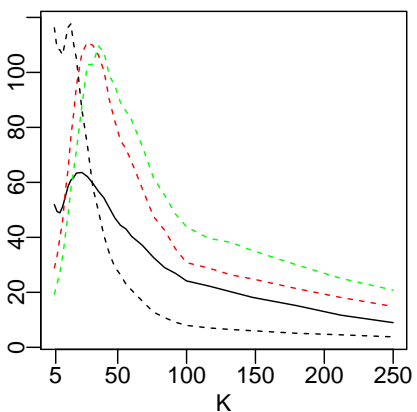

Figure 12: The total computational cost $T_{\mathrm{opt}}^{(\mathcal{X})}$ (left), and the precision per CPU second (right) of the optimal bridge sampling estimators $\hat{\lambda}_{\mathrm{opt}}^{(\mathrm{U})}$ (solid lines, $m=n$ ) and $\hat{\lambda}_{\mathrm{opt}}^{(\mathrm{mix})}$ (dashed lines) with $m=n$ (black), $16 n$ (red), and $32 n$ (green).

$L$ draws and unnecessary computational cost. At this time, we have not been able to obtain any simple rule for constraining the optimal $K$ further. We want $K$ to be large enough to induce sufficient overlap between $\phi$ and $\tilde{p}$, but small enough to limit computational cost, both of which require problem specific knowledge. However, as discussed in the main text, the beauty of Warp-U bridge sampling is that it performs very well even when $\phi_{\text {mix }}$ is not a great fit to $p$.

\section{B.2 Impact of $L$}

Other factors being fixed, larger $L$ on average results in more overlap between $p$ and $\phi_{\text {mix }}$, and hence between $\tilde{p}$ and $\phi$. Consequently, the statistical efficiency of $\hat{\lambda}_{\mathrm{opt}}^{(\mathrm{U})}$ increases with $L$. It follows that if computational cost is not a concern, we should use all of the draws in one half of the draws from $p$ to estimate $\boldsymbol{\zeta}$, and to apply the corresponding Warp- $\mathrm{U}$ transformation to the other half in order to obtain $\hat{\lambda}_{\mathrm{opt}, i}^{(\mathrm{U})}$, for $i=1,2$. However, increasing $L$ above moderate values may yield diminishing returns, even in the case where $p$ is exactly a Normal mixture so that the $f$-divergence between $p$ and $\phi_{\text {mix }}\left(\cdot ; \widetilde{\boldsymbol{\zeta}}_{L}\right)$ tends to zero as $L \rightarrow \infty$.

Figure 13 shows the impact of $L$ on (i) $T_{\mathrm{EM}}$, the computational cost in CPU seconds of estimating $\boldsymbol{\zeta}$; (ii) $T_{\mathrm{opt}}^{(\mathrm{U})}$ and $T_{\mathrm{opt}}^{(\mathrm{mix})}$, the total computational cost in CPU seconds of obtaining $\hat{\lambda}_{\text {opt }}^{(\mathrm{U})}$ and $\hat{\lambda}_{\text {opt }}^{\text {(mix) }}$; and (iii) the RMSE. Intuitively, we should increase the size of the sample for estimating $\zeta$ linearly with $K$, so we compare estimators with different values of $L / K$. In the left panel of Figure 13 we can see that $T_{\mathrm{EM}}$ increases linearly with $L$, for fixed $K$, and therefore so does $T_{\mathrm{opt}}^{(\mathcal{X})}$ (middle panel), because $L$ affects only the EM part. The right panel of Figure 13 shows that RMSE decreases as $L / K$ increases, but the reduction rate becomes very small when $L / K>50$. We obtained similar results (not shown) for the 50-dimension example in Section 4.3. Thus, a reasonable rule of thumb is to set $L=\min (50 K, n / 2)$. 
$T_{\mathrm{EM}}$ in seconds

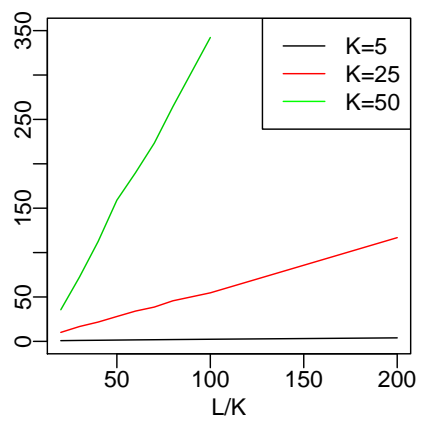

$T_{\mathrm{opt}}^{(\mathcal{X})}$ in seconds

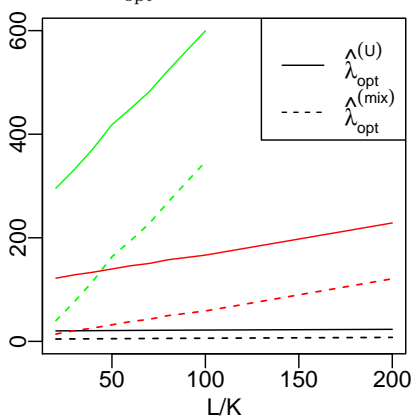

$\log (\mathrm{RMSE})$

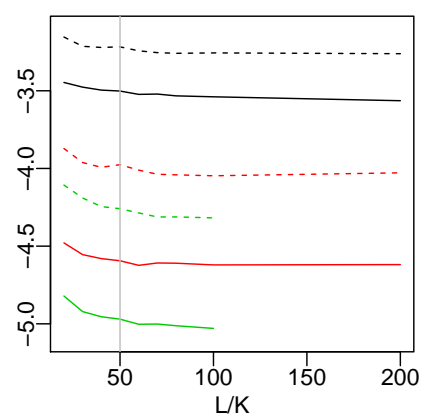

Figure 13: The impact of $L$ on $T_{\mathrm{EM}}$ (left), $T_{\mathrm{opt}}^{(\mathcal{X})}$ (middle), and the log RMSE of $\hat{\lambda}_{\mathrm{opt}}^{(\mathcal{X})}$ (right). Black lines: $K=5$; red lines: $K=25$; green lines: $K=50$. When $K=50$, we can take $L / K$ only up to 100 , because of the restriction that $L \leqslant n / 2$.
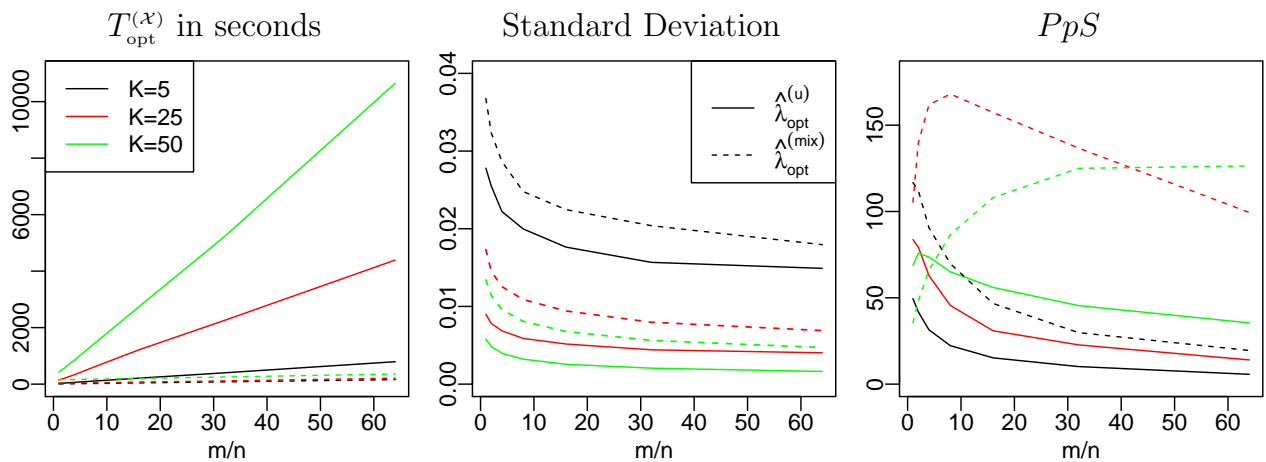

Figure 14: The total CPU time $T_{\mathrm{opt}}^{(\mathcal{X})}$ (left), the standard deviation (middle), and the $P p S$ of $\hat{\lambda}_{\mathrm{opt}}^{(\mathrm{U})}$ (solid lines) and $\hat{\lambda}_{\mathrm{opt}}^{(\mathrm{mix})}$ (dashed lines) with various choices of $m$ for $K=5$ (black), 25 (red), and 50 (green).

\section{B.3 Impact of $m$ and a Comparison of $\hat{\lambda}_{\text {opt }}^{\text {(mix) }}$ and $\hat{\lambda}_{\text {opt }}^{(\mathrm{U})}$}

As with $K$ and $L / K$, larger $m$ improves the precision of the estimators but also increases the computational cost. In the case of $\hat{\lambda}_{\mathrm{opt}}^{\text {(mix) }}$, setting $m>n$ may only increase the computational cost by a small amount and therefore can be a worthwhile trade-off. In particular, when $K$ is large and target evaluations are inexpensive, $T_{\mathrm{EM}}$ will typically dominate $T_{\mathrm{BS}}^{(\mathrm{mix})}$ meaning that the additional computational cost introduced by increasing $m$ is small and likely acceptable given the potential for substantial improvements in statistical efficiency. Indeed, referring back to Figure 12 (left), when $K$ is moderate or large, we see that the difference in the computational cost of $\hat{\lambda}_{\text {opt }}^{\text {(mix) }}$ across the cases $m=n$ (black dashed line), $16 n$ (red dashed line), and $32 n$ (green dashed line), is negligible compared with the total cost $T_{\mathrm{EM}}+T_{\mathrm{BS}}^{(\mathrm{mix})}$. Consequently, $\hat{\lambda}_{\mathrm{opt}}^{(\mathrm{mix})}(16 n)$ and $\hat{\lambda}_{\text {opt }}^{\text {(mix) }}(32 n)$ have larger $\operatorname{PpS}$ than $\hat{\lambda}_{\text {opt }}^{(\mathrm{U})}(n)$ for moderate and large $K$, where $\hat{\lambda}_{\text {opt }}^{(\mathcal{X})}(m)$ denotes the estimator $\hat{\lambda}_{\mathrm{opt}}^{(\mathcal{X})}$ with a specific setting of $m$; see Figure 12 (right). 
Figure 14 further confirms these findings, and demonstrates that increasing $m$ does not offer the same improvements in $P p S$ in the case of $\hat{\lambda}_{\mathrm{opt}}^{(\mathrm{U})}$. Figure 14 (left) shows that both $T_{\mathrm{opt}}^{(\mathrm{U})}$ (solid lines) and $T_{\mathrm{opt}}^{(\mathrm{mix})}$ (dashed lines) grow linearly as $m$ increases from $n$ to $64 n$. Figure 14 (middle) shows that the standard deviation of $\hat{\lambda}_{\mathrm{opt}}^{(\mathcal{X})}$ is inversely related to $m$. Figure 14 (right) shows that the $P p S$ of $\hat{\lambda}_{\mathrm{opt}}^{(\mathrm{U})}$ usually decreases as $m$ increases, which is a result of the high computational cost associated with increases in $m$. However, we again see that $m>n$ can improve the $P p S$ for $\hat{\lambda}_{\mathrm{opt}}^{(\mathrm{mix})}$, because the computational cost of the bridge sampling step, $T_{\mathrm{BS}}^{(\mathrm{mix})}$, is low compared with $T_{\mathrm{EM}}$ and $T_{\mathrm{BS}}^{(\mathrm{U})}$.

Despite the above findings, given a fixed sample of size $n$ from $p$, the best statistical efficiency achieved by $\hat{\lambda}_{\text {opt }}^{(\mathrm{U})}$ is better than that of $\hat{\lambda}_{\text {opt }}^{(\text {mix })}$. The higher computational cost of $\hat{\lambda}_{\text {opt }}^{(\mathrm{U})}$ can be mitigated by parallel computing, for instance, which is a topic for further research. Moreover, in cases where target evaluations are expensive, there will be much less opportunity for improving the $P p S$ of $\hat{\lambda}_{\mathrm{opt}}^{(\mathrm{mix})}$ by increasing $m$ because $T_{\mathrm{EM}}$ will not dominate $T_{\mathrm{BS}}^{(\mathrm{mix})}$. In these cases the superiority of $\hat{\lambda}_{\text {opt }}^{\text {(mix) }}$ in terms of $P p S$ disappears, as confirmed by the example in Section 5 .

To sum up, we recommend using $L=50 K$ draws from $p$ to estimate $\zeta$. The variance of $\hat{\lambda}_{\mathrm{opt}}^{(\mathcal{X})}$ can be effectively reduced by increasing $K$ and/or $m$ up to certain levels. The rates of reduction in variance are different for $K$ and $m$. When $K$ is small, increasing $K$ reduces the variance faster than increasing $m$; when $K$ is large, increasing $m$ is more beneficial for reducing the variance. For the estimator $\hat{\lambda}_{\mathrm{opt}}^{(\mathrm{mix})}$, having a large $m$, e.g., $m=10 n$, is recommended in cases where $T_{\mathrm{BS}}^{(\text {mix })}$ is low compared with $T_{\mathrm{EM}}$.

\section{Efficiency of Diagonal vs. Full Covariance Matrices}

Here we compare the computational and statistical efficiency of using diagonal and full covariance matrices in $\phi_{\text {mix }}$ in (7). The left panel of Figure 15 shows the log RMSE of $\hat{\lambda}_{\mathcal{I}, \text { Diag }}^{(\mathrm{U})}, \hat{\lambda}_{\mathcal{I}, \text { Full }}^{(\mathrm{U})}, \hat{\lambda}_{\mathrm{H}, \text { Diag }}^{(\mathrm{U})}$, and $\hat{\lambda}_{\mathrm{H}, \text { Full }}^{(\mathrm{U})}$. On average, the log RMSE of $\hat{\lambda}_{\mathcal{I}, \text { Diag }}^{(\mathrm{U})}$ (thin solid line) is about $50 \%$ larger than that of $\hat{\lambda}_{\mathcal{I}, \text { Full }}^{(\mathrm{U})}$ (thick solid line). Recall that the $\mathcal{I}$ subscript indicates that an additional independent set of draws from $p$ is used to estimate the covariance matrices, and hence there is no adaptive bias and also more degrees of freedom to estimate the covariance matrices. In such cases, there is a benefit to using the full covariance matrices, because they are accurately estimated. However, when we need to estimate the covariance matrices from the original set of draws from $p$ there is not much advantage to using full covariance matrices; the log RMSE of $\hat{\lambda}_{\mathrm{H}, \mathrm{Diag}}^{(\mathrm{U})}$ (thin dashed line) is only $16.7 \%$ larger than that of $\hat{\lambda}_{\mathrm{H}, \mathrm{Full}}^{(\mathrm{U})}$ (thick dashed line), and the difference diminishes as $K$ increases. This is because, when $K$ is large, over-fitting becomes more serious for the full-matrix model due to the additional $K D(D-1) / 2$ parameters in the model. The diagonal-matrix model, being much more parsimonious, continues to fit the date (i.e., the draws from $p$ ) well and the resulting log RMSE decreases at a stable rate. 
RMSE (log scale)

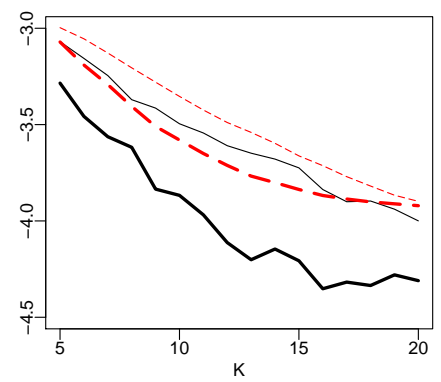

CPU seconds of EM

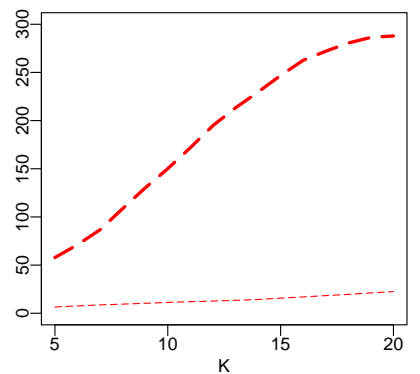

Precision per Second

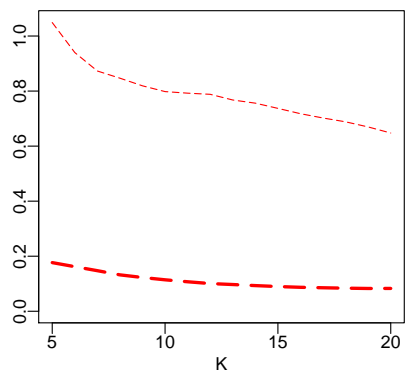

Figure 15: (Left) Log RMSE of different estimators; (middle) CPU seconds used by the EM algorithm; (right) precision per CPU second defined as (1/MSE)/time. (Solid lines) Warp-U bridge sampling with $\widetilde{\boldsymbol{\zeta}}_{\mathcal{I}} ;$ (dashed lines) the average of the two Warp-U bridge sampling estimators with half of the draws fro $p$ used for estimating $\boldsymbol{\zeta}$ and the other half for bridge sampling. (Thin lines) diagonal covariance matrices; (thick lines) full covariance matrices.

Figure 15 (right) shows the CPU seconds for estimating $\boldsymbol{\zeta}$ via EM. On average in this study, it takes 12 times longer to obtain $\phi_{\text {mix }}$ with full covariance matrices than with diagonal covariance matrices, and the difference increases with the dimensionality. In addition, in the bridge sampling step, evaluating $\phi_{\text {mix }}$ with full covariance matrices is much more costly than with diagonal covariance matrices. Therefore, a small loss of statistical efficiency but huge gain in computational efficiency justifies the use of diagonal covariance matrices. Indeed, for reducing RMSE, increasing the number of mixture components $K$ seems to be more effective than using full covariance matrices. This is consistent with our intuition that, for the purposes of increasing distributional overlap, it is more important to increase the chance that our model $\phi_{\text {mix }}$ shares major modes with $p$ than to refine the curvature of $\phi_{\text {mix }}$ at the modes.

\section{Details of GWL implementation}

A complication in specifying the GWL algorithm is identifying a suitable proposal distribution for the Metropolis-Hastings update in its Step 2(i) detailed in Section 5.1. We reproduced the results for the 2 dimensional examples in Liang (2005), but found it difficult to identify a simple proposal that results in comparable performance of the GWL algorithm in our 10 dimensional example. Perhaps a sophisticated method is needed to choose the proposal distribution in examples with more than a few dimensions. For our simulation study, we can avoid this issue by deliberately biasing our comparison in favor of the GWL algorithm (and hence making it harder to show the benefit of our proposal). That is, we adopted the true target density $p$ (which is distinct from the limiting distribution of the GWL algorithm) as a component of the proposal. More precisely, our proposal density is $\frac{40}{41} p_{S}(x)+\frac{1}{41} u(x)$, where $p_{S}$ is $p$ restricted to $S=\bigcup_{i=2}^{r} S_{i}=[-20,20]^{10}$ 
and $u(x)$ is the uniform density on the additional subregion $S_{1}=[20,21] \times[-20,20]^{9}$. Using this proposal does not result in sampling directly from $p\left(\right.$ or $\left.p_{S}\right)$ because the GWL algorithm attempts to sample energy bins uniformly. We verified that the performance of the GWL algorithm is substantially better with this ideal proposal density than under all other proposals that we considered in our investigations (mostly Normal densities with different variances).

The final part of the setup is to define the energy bins $E_{1}, \ldots, E_{r}$, and we again do this in a way that is favorable to the GWL algorithm. We generate 1000 samples from the (ideal) proposal and compute their energies and also evaluate the energy at the true location parameter of each of the 25 skewed- $t$ densities. We then define the energy bins to span the range of these energies in steps of 0.1 (all energies outside this range are put in the end bins). This typically yields around 350 bins but the exact number varies with each run of the algorithm. We briefly experimented with different energy steps but found that 0.1 gave the best results. 